\title{
THE TRANSLATIONAL HULL OF A TOPOLOGICAL SEMIGROUP
}

\author{
BY
}

\author{
J. A. HILDEBRANT, J. D. LAWSON AND D. P. YEAGER
}

\begin{abstract}
This paper is concerned with three aspects of the study of topological versions of the translational hull of a topological semigroup. These include topological properties, applications to the general theory of topological semigroups, and techniques for computing the translational hull. The central result of this paper is that if $S$ is a compact reductive topological semigroup and its translational hull $\Omega(S)$ is given the topology of continuous convergence (which coincides with the topology of pointwise convergence and the compactopen topology in this case), then $\Omega(S)$ is again a compact topological semigroup. Results pertaining to extensions of bitranslations are given, and applications of these together with the central result to semigroup compactifications and divisibility are presented. Techniques for determining the translational hull of certain types of topological semigroups, along with numerous examples, are set forth in the final section.
\end{abstract}

The algebraic theory of the translational hull of a semigroup has been presented extensively in [10] and [11] with earlier material appearing in [3]. References in these works indicate a list of contributors to the field.

Motivation for the study of the translational hull has primarily been its applications to the theory of ideal extensions. For this reason, it appears to the authors of this paper that any coherent theory of ideal extensions of topological semigroups would be based on a topological version of the translational hull. Results of a brief effort in this direction appear in [2], where a topology is assigned to the translational hull. A study of the special case of a compact semilattice is presented in [1].

In this paper we expand the knowledge of topological versions of the translational hull of a topological semigroup in terms of topological properties, applications to the general theory of semigroups, and techniques for computing the translational hull of a given topological semigroup. Whenever feasible, we present results in the more general setting of semitopological semigroups.

Our prime objective in the first section of this paper is to find a reasonable

Received by the editors February 3, 1975 and, in revised form, June 26, 1975.

AMS (MOS) subject classifications (1970). Primary 22A15, $20 \mathrm{M} 20$.

Key words and phrases. Translational hull, topological semigroup, reductive semigroup, net reductive semigroup, bitranslation, topology of continuous convergence, topology of pointwise convergence, extension, semigroup compactification, uniquely divisible semigroup, basis, separating subset. 
topology for the translational hull and develop the basic properties. We propose two candidates, which we believe to be suitable, and indicate that these coincide for a large class of semigroups. The central result of this section is that the translational hull of a compact reductive semigroup is again a compact semigroup. In route to this result, topological analogues of the algebraic notion of reductivity are developed.

The second section is devoted to the study of sufficient conditions for a continuous homomorphism from one topological semigroup to another to induce one between their respective translational hulls.

In the third section we apply the results of the first two sections to the study of semigroup compactifications. Of particular concern is the existence and uniqueness of monomorphic compactifications. The translational hull provides a unifying base from which to derive previously scattered results in [5], [6], and [9] concerning semigroup compactifications.

As an application of the central result of the first section, we obtain a result in the fourth section which appears in [5] and which states that each compact power-cancellative abelian semigroup $S$ can be embedded in a compact uniquely divisible abelian semigroup. The result offered here yields additional information, since the compact uniquely divisible abelian semigroup is a subsemigroup of the translational hull of the original semigroup and the embedding is canonical.

Having observed a deficiency of examples of translational hulls in the literature, we set out to investigate numerous examples and thereby discovered some techniques for computing the translational hull for certain classes of semigroups. The fifth section of this paper is devoted to the presentation of these techniques and examples. Some of the examples reveal information pertaining to the properties of the translational hull, e.g., the translational hull of a compact connected reductive semigroup need not be connected.

The authors are indebted to the referee for several useful and clarifying suggestions concerning continuous convergence, for Lemma 1.0, and for corrections and simplifications in a number of proofs.

1. Topologizing the translational hull. If $S$ is a semigroup, then a function $\lambda: S \rightarrow S$ is called a left translation of $S$ provided $\lambda(x y)=(\lambda x) y$ for each $x, y$ $\in S$, and a function $\rho: S \rightarrow S$ is called a right translation of $S$ provided $(x y) \rho=$ $x(y \rho)$ for each $x, y \in S$. (Here we write $x \rho$ for the image of $x$ under $\rho$.) If $\lambda$ and $\rho$ are left and right translations of $S$, respectively, such that $x(\lambda y)=(x \rho) y$ for each $x, y \in S$, then we say that $\lambda$ and $\rho$ are linked and the linked pair $(\lambda, \rho)$ is called a bitranslation of $S$. If $\omega=(\lambda, \rho)$ is a bitranslation of $S$ and $a \in S$, then we frequently denote $\omega a=\lambda a$ and $a \omega=a \rho$. Observe that for $x, y \in S$ we have $\omega(x y)=(\omega x) y,(x y) \omega=x(y \omega)$, and $x(\omega y)=(x \omega) y$. For an element $a \in S$ we 
use $\lambda_{a}\left[\rho_{a}\right]$ to denote the left [right] translation $x \mapsto a x[x \mapsto x a]$. Notice that $\lambda_{a}$ and $\rho_{a}$ are linked for each $a \in S$, and hence $\omega_{a}=\left(\lambda_{a}, \rho_{a}\right)$ is a bitranslation of $S$. For $a \in S$, the translations $\lambda_{a}$ and $\rho_{a}$ are called inner left and inner right translations, respectively, and $\omega_{a}$ is called an inner bitranslation. If $T$ is a subsemigroup of $S$ and $\omega=(\lambda, \rho)$ is a bitranslation of $S$, then $\omega \mid T$ denotes the pair $(\lambda|T, \rho| T)$ (restrictions). Observe that if $a \in S$ and $I$ is an ideal of $S$, then $\omega_{a} I I$ is a bitranslation of $I$.

In the case that a semigroup $S$ is endowed with a topology and $\omega=(\lambda, \rho)$ is a bitranslation of $S$, we say that $\omega$ is a continuous bitranslation provided $\lambda$ and $\rho$ are both continuous. We will use $\Lambda(S)$ and $P(S)$ to denote the set of all continuous left and right translations of $S$, respectively, and use $\Omega(S)$ to denote the set of all continuous bitranslations of $S$. Observe that $\Lambda(S)$ is a semigroup under composition, $P(S)$ is a semigroup under reverse composition, and $\Omega(S)$ is a subsemigroup of $\Lambda(S) \times P(S)$. The semigroup $\Omega(S)$ is called the translational hull of $S$. Notice that the set $\pi(S)$ of all inner bitranslations of $S$ is a subsemigroup of $\Omega(S)$ and the function $\pi: S \rightarrow \pi(S)$ defined by $\pi(a)=\omega_{a}$ is a homomorphism. The semigroup $\pi(S)$ is called the inner translational hull of $S$ and the homomorphism $\pi: S \rightarrow \pi(S)$ is called the canonical homomorphism. For the purpose of topologizing $\Omega(S)$, we will use $S^{S}$ to denote the set of all continuous functions from $S$ into $S$.

If $S$ is a semigroup endowed with a topology, then the topology of pointwise convergence on $S^{S}$ is defined by saying a net $f_{\alpha}$ converges to $f$ in $S^{S}$ if $f_{\alpha}(x)$ converges to $f(x)$ for each $x \in S$. We denote by $\Lambda_{p}(S)$ and $P_{p}(S)$, the semigroups $\Lambda(S)$ and $P(S)$, respectively, endowed with the relative topology of pointwise convergence on $S$, and by $\Omega_{p}(S)$ the semigroup $\Omega(S)$ with the relative product topology on $\Lambda_{p}(S) \times P_{p}(S)$.

An alternative description of the space $\Omega_{p}(S)$ may be given as follows: Form a product $\Pi\{S \times S\}_{a \in S}$, one factor of $S \times S$ for each element of $S$, endowed with the product topology. Define an injection of $\Omega(S)$ into $\Pi\{S \times S\}_{a \in S}$ by $\omega \mapsto(\omega a, a \omega)$ in the $a$ th coordinate for each $a \in S$. When $\Omega(S)$ is identified as a subset of $\Pi\{S \times S\}_{a \in S}$ in this fashion, then $\Omega_{p}(S)$ is $\Omega(S)$ endowed with the subspace topology.

It is frequently desirable to define a topology on a set from some notion of convergence of nets in that set. (Formally, we would start with a set $F$ and a class $C$ of pairs $\left(\left\{f_{\alpha}\right\}, f\right)$ with $\left\{f_{\alpha}\right\}$ a net in $F$ and $f \in F$, and say that a net $\left\{f_{\alpha}\right\}$ converges to $f$ if $\left(\left\{f_{\alpha}\right\}, f\right)$ is in $C$.) If the notion of convergence satisfies the property that if a net converges to a point, then the net restricted to any cofinal subset of the domain directed set converges to the same point, then a topology may be defined in $F$ by defining a set $A \subset F$ to be closed if and only if whenever $\left\{f_{\alpha}\right\}$ is a net in $A$ which converges to $f$, then $f \in A$. If a net $\left\{f_{\alpha}\right\}$ converges to $f$ by 
definition, then it converges in the topology just defined, but the converse need not necessarily hold. A treatment of these matters appears in [8].

1.0. LEMMA. Let $F$ be a topological space and let $C$ be a class of pairs $\left(\left\{f_{\alpha}\right\}, f\right)$, where $\left\{f_{\alpha}\right\}$ is a net in $F$ and $f \in F$, satisfying: For all $A \subset F, A$ is closed if

$$
\left\{f \in F: \text { there exists }\left\{f_{\alpha}\right\} \subset A \text { with }\left(\left\{f_{\alpha}\right\}, f\right) \in C\right\} \subset A \text {. }
$$

Let $G$ be a space and let $h: F \rightarrow G$. Then $h$ is continuous if $\left(\left\{f_{\alpha}\right\}, f\right) \in C$ implies $\left\{h\left(f_{\alpha}\right)\right\}$ converges to $h(f)$ in $G$.

Proof. Let $B$ be a closed subset of $G$, and let $A=h^{-1}(B)$. If $\left\{f_{\alpha}\right\} \subset A$ and $\left(\left\{f_{\alpha}\right\}, f\right) \in C$, then $\left\{h\left(f_{\alpha}\right)\right\} \subset B$ and $\left\{h\left(f_{\alpha}\right)\right\}$ converges to $h(f)$. Since $B$ is closed, $h(f) \in B$. Hence $f \in A$. Thus by hypothesis, $A$ is closed. Hence $h$ is continuous.

If $X$ and $Y$ are topological spaces, let $Y^{X}$ denote the space of all continuous functions from $X$ into $Y$. We say that a net $f_{\alpha}$ in $Y^{X}$ converges continuously to $f$ in $Y^{X}$ if the product net $f_{\alpha}\left(x_{\beta}\right)$ converges to $f(x)$ for each net $x_{\beta}$ converging to $x$ in $X$. The topology of continuous convergence on $Y^{X}$ is defined by saying a set $A$ is closed if and only if whenever a net $f_{\alpha}$ in $A$ converges continuously to some $f$ in $Y^{X}$, then $f \in A$.

Note that if $Y$ is Hausdorff, then the topology of continuous convergence on $Y^{X}$ is Hausdorff since (using 1.0) the identity function is continuous from $Y^{X}$ with the topology of continuous convergence into $Y^{X}$ with the topology of pointwise convergence. Observe also that if $C=\left\{\left(\left\{f_{\alpha}\right\}, f\right)\right.$ : $f$ converges continuously to $f\}$, then $C$ determines the topology of continuous convergence on $Y^{X}$.

In the terminology of R. Arens and J. Dugundji [0] a topology $T$ on $Y^{X}$ is

(a) proper if continuous convergence implies convergence relative to $T$, and

(b) admissible if convergence relative to $T$ implies continuous convergence.

In this language, the topology of continuous convergence is proper and may be characterized as the strongest (i.e., most open sets) proper topology on $Y^{X}$. However, it need not be admissible. If $X$ is locally compact Hausdorff, then the topology of continuous convergence is precisely the compact open topology and is both proper and admissible (see [0]).

We specialize now to the case that $S$ is a semigroup endowed with a topology. We define topologies on $\Lambda(S), P(S), \Omega(S)$, and $\Omega(S) \times \Omega(S)$ by first defining convergence of nets. A net $\lambda_{\alpha}\left(\rho_{\alpha}\right)$ of continuous left (right) translations converges continuously to $\lambda(\rho)$ if they converge continuously as functions. A net $\omega_{\alpha}=\left(\lambda_{\alpha}, \rho_{\alpha}\right)$ of continuous bitranslations converges continuously to $\omega=$ $(\lambda, \rho)$ if $\lambda_{\alpha}$ converges continuously to $\lambda$ and $\rho_{\alpha}$ converges continuously to $\rho$, 
and a net $\left(\tau_{\alpha}, \omega_{\alpha}\right)$ of ordered pairs of continuous bitranslations converges continuously to $(\tau, \omega)$ if $\tau_{\alpha}$ converges continuously to $\tau$ and $\omega_{\alpha}$ converges continuously to $\omega$. The topology of continuous convergence is defined in $\Lambda(S), P(S)$, $\Omega(S)$, and $\Omega(S) \times \Omega(S)$, respectively, by declaring a set closed if and only if whenever a net in the set converges continuously to some point, then the point is also in the set. These semigroups endowed with the topology of continuous convergence are denoted $\Lambda_{c}(S), P_{c}(S), \Omega_{c}(S)$, and $\Omega_{c}(S) \times{ }_{c} \Omega_{c}(S)$, respectively. Note that $\Omega_{c}(S)$ is not necessarily the topology that $\Omega(S)$ inherits as a subset of $\Lambda_{c}(S) \times P_{c}(S)$ nor is $\Omega_{c}(S) \times{ }_{c} \Omega_{c}(S)$ necessarily the product topology, but that both of these properties hold if the topologies of $\Lambda_{c}(S)$ and $P_{c}(S)$ are admissible (in particular, if $S$ is locally compact Hausdorff). If $\Omega_{c}(S)$ is admissible, then $\Omega_{c}(S) \times{ }_{c} \Omega_{c}(S)$ is the product topology.

1.1. Proposition. Let $S$ be a semigroup on a topological space. Then multiplication on $\Omega_{p}(S)$ is separately continuous and multiplication on $\Omega_{c}(S)$ is continuous from $\Omega_{c}(S) \times{ }_{c} \Omega_{c}(S)$ into $\Omega_{c}(S)$. Hence if the topology of continuous convergence on $\Omega_{c}(S)$ is admissible, then multiplication on $\Omega_{c}(S)$ is jointly continuous.

Proof. Suppose that $\omega_{\alpha}$ is a net in $\Omega_{p}(S)$ converging to $\omega$ and let $\tau \in$ $\Omega_{p}(S)$. Then for each $x \in S$, we have that $\omega_{\alpha} x$ converges to $\omega x$, and since $\tau$ is continuous, $\tau \omega_{\alpha} x$ converges to $\tau \omega x$. Now, since $\omega_{\alpha}$ converges pointwise to $\omega, \omega_{\alpha}(\tau x)$ converges to $\omega(\tau x)$ for all $x \in S$, and hence $\omega_{\alpha} \tau$ converges to $\omega \tau$. Similar arguments on the right complete the proof that multiplication on $\Omega_{p}(S)$ is separately continuous.

To show multiplication is continuous from $\Omega_{c}(S) \times_{c} \Omega_{c}(s)$ to $\Omega_{c}(S)$, we apply Lemma 1.0. Let $\left(\omega_{\alpha}, \tau_{\alpha}\right)$ converge continuously to $(\omega, \tau)$. Then $\omega_{\alpha}$ converges continuously to $\omega$ and $\tau_{\alpha}$ converges continuously to $\tau$. Hence if $x_{\beta}$ converges to $x$ in $S$, then $\tau_{\alpha} x_{\beta}$ converges to $\tau x$, and hence $\omega_{\alpha}\left(\tau_{\alpha} x_{\beta}\right)$ converges to $\omega(\tau x)=(\omega \tau) x$. A similar argument holds on the right. Hence $\omega_{\alpha} \tau_{\alpha}$ converges continuously to $\omega \tau$, and hence converges in the topology of continuous convergence, $\Omega_{c}(S)$.

A semigroup on a Hausdorff space is called a semitopological semigroup provided multiplication is separately continuous and a topological semigroup if multiplication is jointly continuous.

\subsection{Proposition. Let $S$ be a topological [semitopological] semigroup.} Then the canonical homomorphism $\pi: S \rightarrow \Omega_{c}(S)\left[\Omega_{p}(S)\right]$ is continuous.

Proof. Suppose that $S$ is a topological semigroup and suppose $a_{\alpha}$ is a net in $S$ converging to $a$. Then for each net $x_{\beta}$ converging to $x$ in $S$, we have $\omega_{a_{\alpha}} x_{\beta}=$ $a_{\alpha} x_{\beta}$ converging to $a x=\omega_{a} x$ and similarly on the right. It follows that $\pi\left(a_{\alpha}\right)$ 
converges to $\pi(a)$ in the topology of $\Omega_{c}(S)$, and hence $\pi: S \rightarrow \Omega_{c}(S)$ is continuous.

A similar argument applies for the semitopological case.

Various notions of reductivity have played an important role in the study of translational hulls. A semigroup $S$ is said to be left [right] reductive if $x a=$ $x b[a x=b x]$ for all $x \in S$ implies $a=b$, and reductive if $S$ is both left and right reductive. We say that a semigroup $S$ is weakly reductive if $x a=x b$ and $a x=b x$ for all $x \in S$ implies $a=b$.

For the topological setting we need appropriate topological notions of reductivity. We use the notation $a_{\alpha} \rightarrow a$ to mean that the net $a_{\alpha}$ converges to $a$.

A semitopological semigroup $S$ is said to be left [right] net reductive if $x a_{\alpha}$ $\rightarrow x a\left[a_{\alpha} x \rightarrow a x\right]$ for all $x \in S$ implies that $a_{\alpha} \rightarrow a$, and $S$ is said to be net reductive if $S$ is both left and right net reductive. We say that $S$ is weakly net reductive if $x a_{\alpha} \rightarrow x a$ and $a_{\alpha} x \rightarrow a x$ for all $x \in S$ implies that $a_{\alpha} \rightarrow a$.

A semitopological semigroup $S$ is said to be weakly bi-net reductive if for a net $a_{\alpha}$ in $S$ and $a \in S$, the condition that $x_{\beta} a_{\alpha} \rightarrow x a$ and $a_{\alpha} x_{\beta} \rightarrow a x$ for each $x_{\beta} \rightarrow x$ in $S$ implies that $a_{\alpha} \rightarrow a$. The notions of right bi-net reductive, left bi-net reductive, and bi-net reductive are defined analogously.

1.3. Proposition. In a semitopological semigroup $S$ net reductivity implies bi-net reductivity which in turn implies reductivity. Analogous statements hold for right, left, and weak reductivity.

Proof. We give a proof for the case of left reductivity.

Suppose that $S$ is left net reductive. Let $\left\{a_{\alpha}\right\}$ be a net in $S$ and $a \in S$ such that $x_{\beta} a_{\alpha} \rightarrow x a$ for each $x_{\beta} \rightarrow x$ in $S$. Then, in particular, for the constant net $x_{\beta}=x$, we have $x a_{\alpha} \rightarrow x a$. In view of the fact that $S$ is left net reductive, we see that $a_{\alpha} \rightarrow a$, and hence $S$ is left bi-net reductive.

Assume now that $S$ is left bi-net reductive and suppose that $x a=x b$ for all $x \in S$. We show that the constant net consisting of $a$ converges to $b$ (yielding that $a=b$, since $S$ is Hausdorff). If $x_{\beta} \rightarrow x$, then $x_{\beta} a \rightarrow x a=x b$ by separate continuity of multiplication. Since $S$ is left bi-net reductive, the constant net consisting of $a$ converges to $b$.

It is well known in the algebraic case that the canonical homomorphism $\pi: S \rightarrow \Omega(S)$ is an isomorphism into if and only if $S$ is weakly reductive [3] . The next proposition is a topological analogue of this result.

1.4. Proposition. Let $S$ be a semitopological [topological] semigroup. Then the canonical homomorphism $\pi: S \rightarrow \Omega_{p}(S)\left[\Omega_{c}(S)\right]$ is both an isomorphism and homeomorphism into if and only if $S$ is weakly net [bi-net] reductive.

Proof. The proof in the topological semigroup case is essentially in [2]. 
Suppose that $S$ is a semitopological semigroup which is weakly net reductive. By 1.2, we have that $\pi: S \rightarrow \Omega_{p}(S)$ is a continuous homomorphism. From 1.3, we have that $S$ is weakly reductive, and hence $\pi$ is a monomorphism. Suppose now that $\omega_{a_{\alpha}}$ is a net in $\pi_{p}(S)\left(\pi(S)\right.$ with the relative topology of $\left.\Omega_{p}(S)\right)$ converging to $\omega_{a}$. Then for each $x \in S$, we have $\omega_{a_{\alpha}} x=a_{\alpha} x$ converges to $\omega_{a} x=$ $a x$ and similarly $x a_{\alpha}$ converges to $x a$. By weak net reductivity $a_{\alpha}$ converges to $a$. It follows that $\pi^{-1}: \pi(S) \rightarrow S$ is continuous and $\pi$ is a homeomorphism into $\Omega_{p}(S)$.

Conversely suppose that $\pi: S \rightarrow \Omega_{p}(S)$ is a homeomorphism into. Suppose that $a \in S$ and $a_{\alpha}$ is a net in $S$ such that $x a_{\alpha} \rightarrow x a$ and $a_{\alpha} x \rightarrow a x$ for all $x \in$ $S$. By definition of the topology of $\Omega_{p}(S)$, we have $\omega_{a_{\alpha}} \rightarrow \omega_{a}$ in $\Omega_{p}(S)$. Since $\pi$ is a homeomorphism, we have that $a_{\alpha} \rightarrow a$.

Before proceeding to our next proposition, let us make an observation that will be used throughout this and other sections without further reference. If $S$ is a reductive abelian semigroup, then

$$
\Lambda(S)=P(S) \text { and } \Omega(S)=\Delta(\Lambda(S) \times P(S))=\Delta
$$

is an abelian semigroup (see [11]).

1.5. Proposition. Let $S$ be a reductive abelian semigroup endowed with a topology. Then first projection of $\Omega_{c}(S)\left[\Omega_{p}(S)\right]$ is an isomorphism and a homeomorphism onto $\Lambda_{c}(S)\left[\Lambda_{p}(S)\right]$, and second projection of $\Omega_{c}(S)\left[\Omega_{p}(S)\right]$ is an isomorphism and a homeomorphism onto $P_{c}(S)\left[P_{p}(S)\right]$.

Proof. The fact that these projections are isomorphisms is proved in [10]. The fact that $\pi_{i}$ is a homeomorphism $(i=1,2)$ follows from the above remarks, 1.0 , and the diagram:

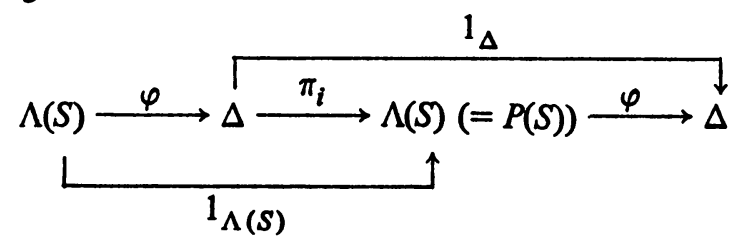

where $\varphi(\lambda)=(\lambda, \lambda)$. We have that $\varphi$ is a continuous inverse for $\pi_{i}$. This com. pletes the proof of the proposition.

The parallel development given for semitopological and topological semigroups ends at this point. For the remainder of this section we treat peculiarities of each situation.

1.6. Proposition. Each bitranslation of a net reductive semitopological semigroup $S$ is continuous, i.e., $\Omega(S)=\Omega\left(S_{d}\right)$, where $S_{d}$ is $S$ endowed with the discrete topology. 
Proof. Let $\omega$ be a bitranslation of $S$ and let $y_{\alpha}$ be a net in $S$ converging to $y$. Then for each $x \in S$, we have $x\left(\omega y_{\alpha}\right)=(x \omega) y_{\alpha} \rightarrow(x \omega) y=x(\omega y)$. Since $S$ is net reductive, $\omega y_{\alpha} \rightarrow \omega y$ and similarly $y_{\alpha} \omega \rightarrow y \omega$. It follows that $\omega$ is continuous.

1.7. Proposition. Let $S$ be a net reductive topological semigroup. If $\omega_{\alpha}$ is a net in $\Omega(S)$ and $\omega \in \Omega(S)$, then $\omega_{\alpha}$ converges pointwise to $\omega$ if and only if $\omega_{\alpha}$ converges continuously to $\omega$. Hence, in this case, $\Omega_{p}(S)=\Omega_{c}(S)$, the topology of continuous convergence is admissible, and $\Omega_{c}(S)$ is a topological semigroup.

Proof. The identity function on $\Omega(S)$ is continuous from $\Omega_{c}(S)$ to $\Omega_{p}(S)$ in view of 1.0 .

Suppose $\omega_{\alpha} \rightarrow \omega$ in $\Omega_{p}(S)$, and let $x_{\beta} \rightarrow x$ in $S$. Then for each $y \in S$, $y\left(\omega_{\alpha} x_{\beta}\right)=\left(y \omega_{\alpha}\right) x_{\beta} \rightarrow(y \omega) x=y(\omega x)$.

Since $S$ is net reductive, we have $\omega_{\alpha} x_{\beta} \rightarrow \omega x$; similarly, $x_{\beta} \omega_{\alpha} \rightarrow x \omega$, so that $\omega_{\alpha}$ converges continuously to $\omega$. Hence $\Omega_{p}(S)=\Omega_{c}(S)$.

If $\omega_{\alpha}$ converges to $\omega$ in $\Omega_{c}(S)$, then by the preceding $\omega_{\alpha}$ converges pointwise to $\omega$, and hence $\omega_{\alpha}$ converges continuously to $\omega$. Thus $\Omega_{c}(S)$ is admissible. By 1.1, $\Omega_{c}(S)$ is a topological semigroup, and the proof of the proposition is complete.

We turn now to the case that will occupy most of our attention in the remainder of this paper, the compact case, and develop the basic results for that case. The next proposition is a partial converse to 1.3.

\subsection{Proposition. Let $S$ be a compact semitopological semigroup and} let $T$ be a dense subsemigroup of $S$. If $S$ is reductive, then $T$ is net reductive. Analogous statements hold for right, left, and weak reductivity.

Proof. We prove only the case for left reductivity since the others are similar. Let $y_{\alpha}$ be a net in $T$ and $y \in T$ such that $x y_{\alpha} \rightarrow x y$ for all $x \in T$. Let $z$ be a cluster point of the net $y_{\alpha}$ in $S$. Then for each $x \in T$, the net $x y_{\alpha}$ clusters to $x z$ in $S$ and converges to $x y$. Hence for all $x \in T$, we have $x z=x y$. Since $T$ is dense in $S$ and multiplication is separately continuous, we have $x z=$ $x y$ for all $x \in S$. Since $S$ is left reductive, we have $z=y$, so that $y_{\alpha} \rightarrow y$.

1.9. REMARK. It is useful to observe that if $S$ is a reductive semigroup and $f$ and $g$ are a linked pair of functions from $S$ into $S$, then $(f, g)$ is a bitranslation of $S$ (see [10]). To see that $f$ is a left translation let $x, y \in S$. Then for each $t \in S$, we have $t[(f x) y]=[t(f x)] y=[(t g) x] y=(t g)(x y)=t f(x y)$. Using left reductivity, we see that $(f x) y=f(x y)$, and $f$ is a left translation. A similar argument works to show that $g$ is a right translation of $S$, and hence $(f, g)$ is a bitranslation of $S$. 
1.10. Proposition. Let $S$ be a compact reductive semitopological semigroup. Then $\Omega_{p}(S)$ is a compact semitopological semigroup.

Proof. Embed $\Omega(S)$ in $\Pi\{S \times S\}_{a \in S}$ by $\omega \mapsto(\omega a, a \omega)$ in the $a$ th coordinate for each $a \in S$. We show that $\Omega(S)$ embedded in this way is a closed subset of $\Pi\{S \times S\}_{a \in S}$.

Let $\omega_{\alpha}$ be a net in $\Omega(S)$ converging to an element $\omega$ of $\Pi\{S \times S\}_{a \in S}$. We may consider $\omega$ to be a bifunction by defining $\omega x$ to be the first entry in the $x$ th coordinate of $\omega$ and $x \omega$ the second. To see that $\omega$ is a linked pair, let $x, y \in S$. Then $x(\omega y)=x\left(\lim \omega_{\alpha} y\right)=\lim x\left(\omega_{\alpha} y\right)=\lim \left(x \omega_{\alpha}\right) y=\left(\lim x \omega_{\alpha}\right) y$ $=(x \omega) y$. In view of 1.9 and the fact that $S$ is reductive, we have that $\omega$ is a bitranslation of $S$. In view of 1.6 and 1.8 , we see that $\omega$ is continuous and thus $\Omega(S)$ is closed in $\Pi\{S \times S\}_{a \in S}$. Since $\Omega(S)$ with the subspace topology is just $\Omega_{p}(S)$, we have that $\Omega_{p}(S)$ is a closed subset of a compact space and hence compact. By 1.1 multiplication on $\Omega_{p}(S)$ is separately continuous.

Combining some of the results of this section we obtain the following:

1.11. Corollary. Let $S$ be a compact reductive topological semigroup. Then $\Omega_{p}(S)=\Omega_{c}(S)$ consists of all bitranslations of $S$ and is a compact topological semigroup. Furthermore, the canonical homomorphism $\pi: S \rightarrow \Omega_{c}(S)$ is a topological isomorphism onto the ideal of inner bitranslations. If, in addition, $S$ is abelian, then $\Omega_{c}(S)=\Omega_{p}(S), \Lambda_{c}(S)=\Lambda_{p}(S)$, and $P_{c}(S)=P_{p}(S)$ are all topologically isomorphic compact abelian topological semigroups.

We close this section with a few historical comments. T. Bowman [1] anticipated some of the central results of this section by showing that any left translation on a compact topological semilattice is continuous, and that the set of all left translations formed a compact topological semilattice with the compact-open topology (= the topology of continuous convergence for locally compact semigroups). Notice that semilattices are reductive, and hence Bowman's result follows from 1.11 .

F. Christoph [2] suggested topologizing $\Omega(S)$ with the topology of continuous convergence and introduced the first notions of net reductivity. One-half of 1.4 is his result.

Our starting point for this paper was the central result of 1.11 that the translational hull of a compact reductive semigroup is again a compact semigroup with the compact-open topology, and was motivated by the technique introduced by $M$. Friedberg in [4].

2. Extending bitranslations. Unfortunately the assignment of $\Omega(S)$ to $S$ is not functorial, i.e., if $f: S \rightarrow T$ is a homomorphism there is in general no natural way to define $\Omega(f): \Omega(S) \rightarrow \Omega(T)$ to commute with the canonical 
homomorphisms of $S$ and $T$. This section is devoted to pointing out some special situations where this is possible.

If $T$ is a subsemigroup of a semigroup $S$ and $\omega$ is a bitranslation of $T$, then a bitranslation $\omega^{\prime}$ of $S$ is called an extension of $\omega$ to $S$ provided $\omega^{\prime} \mid T=\omega$.

2.1. Proposition. Let $S$ be a compact reductive semitopological semigroup and let $T$ be a dense subsemigroup of $S$. Then each bitranslation of $T$ can be extended to a unique continuous bitranslation of $S$.

Proof. Let $\omega=(\lambda, \rho)$ be a bitranslation of $T$. We identify $\lambda$ with its graph, so that $\lambda \subset T \times T \subset S \times S$. Let $\lambda^{*}$ be the closure of $\lambda$ in $S \times S$. We show that $\lambda^{*}$ is a function. Suppose that $\left(x, y_{1}\right)$ and $\left(x, y_{2}\right)$ are in $\lambda^{*}$. Then there exist nets $x_{\alpha}$ and $x_{\beta}$ in $T$ converging to $x$ such that $\lambda x_{\alpha} \rightarrow y_{1}$ and $\lambda x_{\beta}$ $\rightarrow y_{2}$. For each $t \in T$, we have $t y_{1}=t\left(\lim \lambda x_{\alpha}\right)=\lim t\left(\lambda x_{\alpha}\right)=\lim (t \rho) x_{\alpha}=$ $(t \rho) \lim x_{\alpha}=(t \rho) x$. Similarly, $t y_{2}=(t \rho) x$, and so $t y_{1}=t y_{2}$. By reductivity, it follows that $y_{1}=y_{2}$, and $\lambda^{*}$ is a function. Since $\lambda^{*}$ is compact, we have that $\pi_{1}\left(\lambda^{*}\right)$ is compact, where $\pi_{1}: S \times S \rightarrow S$ is first projection. Since $\pi_{1}\left(\lambda^{*}\right)$ contains $T$ and $T$ is dense in $S$, we obtain that $\pi_{1}\left(\lambda^{*}\right)=S$ and $S=$ domain $\lambda^{*}$. Finally, $\lambda^{*}$ is continuous, since $S$ is compact and $\lambda^{*}$ has closed graph.

We similarly define $\rho^{*}$ to be the closure of $\rho$ in $S \times S$, and define $\omega^{*}=$ $\left(\lambda^{*}, \rho^{*}\right)$. Then $\omega^{*}$ is a continuous bifunction whose restriction to $T$ is the bitranslation $\omega$. It is straightforward to verify that if a continuous bifunction restricted to a dense subsemigroup is a bitranslation, then it is itself a bitranslation.

We turn now to a result (2.3) which will be useful in later sections.

2.2. LEMMA. Let $S$ be a semigroup, $T$ a reductive semigroup, $f: S \rightarrow T$ a surmorphism, and $\omega$ a bitranslation of $S$. Then there exists a unique bitranslation $\omega^{\prime}$ of $T$ such that $\omega^{\prime} f(x)=f(\omega x)$ and $f(x) \omega^{\prime}=f(x \omega)$ for each $x \in S$.

Proof. For $y \in T$, we define $\omega^{\prime} y=f(\omega x)$, where $y=f(x)$. To see that $\omega^{\prime} y$ is well defined suppose that $y=f(x)=f\left(x^{\prime}\right)$. Then for $t \in T$, we have $t=f(z)$ for some $z \in S$, so that $t f(\omega x)=f(z) f(\omega x)=f(z(\omega x))=f((z \omega) x)=$ $f(z \omega) f(x)=f(z \omega) f\left(x^{\prime}\right)=f\left((z \omega) x^{\prime}\right)=f\left(z\left(\omega x^{\prime}\right)\right)=f(z) f\left(\omega x^{\prime}\right)=t f\left(\omega x^{\prime}\right)$. Since $T$ is reductive, we have that $f(\omega x)=f\left(\omega x^{\prime}\right)$. We also define $y \omega^{\prime}=f(x \omega)$, where $y=f(x)$, and employ a similar argument to show that $y \omega^{\prime}$ is well defined.

To see that $\omega^{\prime}$ is a bitranslation of $T$, we need only demonstrate the linking property by virtue of 1.9. Let $a, b \in T$ and $a^{\prime}$ and $b^{\prime}$ elements of $S$ such that $a=f\left(a^{\prime}\right)$ and $b=f\left(b^{\prime}\right)$. Then $a\left(\omega^{\prime} b\right)=f\left(a^{\prime}\right)\left(\omega^{\prime} f\left(b^{\prime}\right)\right)=f\left(a^{\prime}\right) f\left(\omega b^{\prime}\right)=$ $f\left(a^{\prime}\left(\omega b^{\prime}\right)\right)=f\left(\left(a^{\prime} \omega\right) b^{\prime}\right)=f\left(a^{\prime} \omega\right) f\left(b^{\prime}\right)=\left(f\left(a^{\prime}\right) \omega^{\prime}\right) b=\left(a \omega^{\prime}\right) b$.

If $S$ and $T$ are semitopological semigroups, we say that a function $f: S \rightarrow$ $T$ is dense provided $f(S)$ is dense in $T$. 
Combining 2.1 and 2.2, we obtain:

2.3. Proposition. Let $S$ be a semitopological semigroup, $T$ a compact reductive semitopological semigroup, $f: S \rightarrow T$ a dense continuous homomorphism, and $\omega$ a bitranslation of $S$. Then there exists a unique continuous bitranslation $\omega^{\prime}$ of $T$ such that $\omega^{\prime} f(x)=f(\omega x)$ and $f(x) \omega^{\prime}=f(x \omega)$ for all $x \in S$.

2.4. Proposition. Let $S$ be a semitopological semigroup, $T$ a compact reductive semitopological semigroup, and $f: S \rightarrow T$ a dense continuous homomorphism. Then there exists a unique continuous homomorphism $\Omega(f): \Omega_{p}(S)$ $\rightarrow \Omega_{p}(T)$ such that the following diagrams commute:
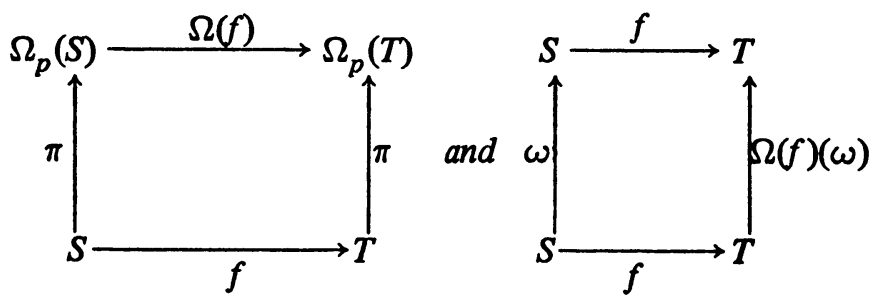

for each $\omega \in \Omega(S)$.

Proof. For $\omega \in \Omega(S)$, we define $\Omega(f)(\omega)$ to be the unique bitranslation $\omega^{\prime}$ guaranteed by 2.3 which makes the second diagram commute. To see that the first diagram commutes, observe that for $x \in S$, we have $\Omega(f)\left(\omega_{x}\right)=\omega_{f(x)}$.

For the purpose of demonstrating that $\Omega(f)$ is continuous, let $\omega_{\alpha} \rightarrow \omega$ in $\Omega_{p}(S)$. Then for each $x \in S$, we have $\omega_{\alpha} x \rightarrow \omega x$, and hence $f\left(\omega_{\alpha} x\right) \rightarrow$ $f(\omega x)$. If $\omega_{\alpha}^{\prime}=\Omega(f)\left(\omega_{\alpha}\right)$ and $\omega^{\prime}=\Omega(f)(\omega)$, then $\omega_{\alpha}^{\prime}(f(x)) \rightarrow \omega^{\prime}(f(x))$ for all $x \in S$, and similarly $(f(x)) \omega_{\alpha}^{\prime} \rightarrow(f(x)) \omega^{\prime}$ for all $x \in S$.

From $1.10, \Omega_{p}(T)$ is compact and so $\left\{\omega_{\alpha}^{\prime}\right\}$ clusters to some $\tau \in \Omega_{p}(T)$. For each $x \in S$, the net $\left\{\omega_{\alpha}^{\prime}(f(x))\right\}$ clusters to $\tau(f(x))$, and hence $\tau(f(x))=$ $\omega^{\prime}(f(x))$. Since $f$ is dense, $\tau t=\omega^{\prime} t$ for each $t \in T$. Similarly, $t \tau=t \omega^{\prime}$ for each $t \in T$, so that $\tau=\omega^{\prime}$. It follows that $\left\{\omega_{\alpha}^{\prime}\right\} \rightarrow \omega^{\prime}$, and $\Omega(f)$ is continuous.

That $\Omega(f)$ is a homomorphism follows immediately from 2.3 and the commutative diagram:

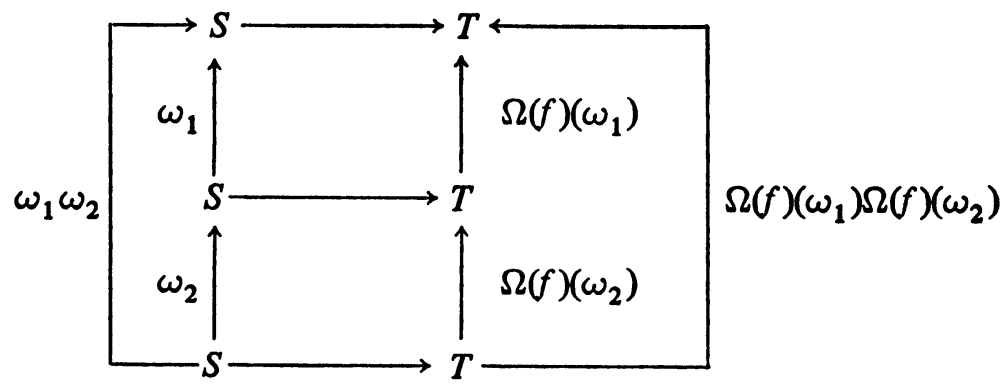

This concludes the proof of the proposition. 
3. Semigroup compactifications. In this section we give a further development of the relation between a semigroup and its translational hull with an eye toward applications to semigroup compactifications.

A compactification of a semitopological semigroup $S$ is a pair $(T, f)$ where $T$ is a compact semitopological semigroup and $f$ is a continuous dense homomorphism from $S$ into $T$. By a slight abuse of notation we sometimes refer to $T$ as the compactification. The next proposition introduces the type of semigroup we will be concerned with in this section.

3.1. Proposition. Let $S$ be a semitopological semigroup. If $S$ is the union of compact left ideals and also the union of compact right ideals, then for any compactification $(T, f)$ of $S, f(S)$ is an ideal in $T$. On the other hand, if $S$ is topologically isomorphic to an ideal of a compact semitopological semigroup, then $S$ is a union of compact right ideals and a union of compact left ideals.

Proof. Suppose $S$ is the union of compact left ideals, $S=\bigcup\{L: L \in L\}$. For each $L \in L, f(L)$ is compact, hence closed. For $x \in S$, there exists $L \in L$ such that $x \in L$. We have

$$
T \cdot f(x)=f(S)^{*} \cdot f(x) \subset(f(S) \cdot f(x))^{*}=f(S x)^{*} \subset f(L)^{*}=f(L) \subset f(S) .
$$

Hence $f(S)$ is a left ideal. Similarly $f(S)$ is a right ideal, and thus an ideal.

Suppose now that $S$ is (topologically isomorphic to) an ideal of a compact semitopological semigroup $T$. Then $S=\bigcup\{T x \cup\{x\}: x \in S\}$, and hence is the union of compact left ideals. Similarly $S$ is the union of compact right ideals.

The first problem we consider is that of finding sufficient conditions for a semigroup $S$ to have a compactification $(T, f)$ where $f$ is a monomorphism. To this end we prove a variant of Proposition 1.10.

3.2. Proposition. Let $S$ be a semitopological semigroup which is globally idempotent $\left(S^{2}=S\right)$, reductive, weakly net reductive, the union of compact left ideals, and the union of compact right ideals. Then $\Omega_{p}(S)$ is compact.

Before proving this proposition, we need the following lemma.

3.3. LEMMA. Let $S$ be a semitopological semigroup which is reductive, weakly net reductive, the union of compact left ideals, and the union of compact right ideals. Then $S$ is net reductive.

Proof. We first show $S$ is left net reductive. Let $x \in S$ and let $x_{\alpha}$ be a net in $S$ such that for all $y \in S, y x_{\alpha}$ converges to $y x$. Since $S$ is weakly net reductive, to show $x_{\alpha}$ converges to $x$ it suffices to show that $x_{\alpha} y$ converges to $x y$ for all $y \in S$. 
Fix $y \in S$, and let $z$ be a cluster point of $x_{\alpha} y$. Then for any $t \in S, t\left(x_{\alpha} y\right)$ clusters to $t z$. Also $\left(t x_{\alpha}\right) y$ converges to $(t x) y=t(x y)$. Hence $t z=t(x y)$ for all $t \in S$; by reductivity $z=x y$. Since $x_{\alpha} y$ is a net in the compact space $(S y)^{*}$ having only the cluster point $x y$, we have that $x_{\alpha} y$ converges to $x y$. Hence $S$ is left net reductive. Similarly $S$ is right net reductive.

Proof of Proposition 3.2. Let $s \in S$. We show $\{\omega s: \omega \in \Omega(S)\}^{*}$ is compact. Let $p, q \in S$ such that $s=p q$. For any $\omega \in \Omega(S), \omega s=\omega(p q)=$ $(\omega p) q \subset(S q)^{*}$, and $(S q)^{*}$ is compact since $S$ is the union of compact left ideals. Similarly $\{s \omega: \omega \in \Omega(S)\}^{*}$ is compact.

Now, as in the proof of $1.10, \Omega_{p}(S)$ is closed in $S^{S} \times S^{S}$ and contained in the compact set $\Pi\left\{A_{s}: s \in S\right\} \times \Pi\left\{B_{s}: s \in S\right\}$, where $A_{s}=\{\omega s: \omega \in \Omega(S)\}^{*}$ and $B_{s}=\{s \omega: \omega \in \Omega(S)\}^{*}$, and it follows that $\Omega_{p}(S)$ is compact.

\subsection{COROLlary. Let $S$ be a topological semigroup which is globally} idempotent, reductive, weakly net reductive, the union of compact left ideals, and the union of compact right ideals. Then every bitranslation on $S$ is continuous, $\Omega_{c}(S)=\Omega_{p}(S)$ is a compact topological semigroup and $\pi: S \rightarrow \Omega_{c}(S)$ is a homeomorphism onto $\pi(S)$.

Proof. This follows immediately from Lemma 3.3 and Propositions 3.2, 1.4, 1.6, 1.7.

\subsection{Corollary. Let $S$ be a semitopological [topological] semigroup} which is globally idempotent, reductive, the union of compact left ideals, and the union of compact right ideals. Then there exists a weakly reductive compactification $(T, f)$ such that $f$ is a monomorphism [and $T$ is a topological semigroup].

Proof. By $1.2 \pi: S \rightarrow \pi(S)$ is continuous, and $\pi$ is an isomorphism since $S$ is reductive.

We show that $\pi(S)$ with the subspace topology from $\Omega_{p}(S)$ is weakly net reductive. Let $\omega_{x} \in \pi(S)$ and let $\omega_{x_{\alpha}}$ be a net in $\pi(S)$ such that for all $\omega_{y}$ in $\pi(S), \omega_{y} \omega_{x_{\alpha}}$ converges to $\omega_{y} \omega_{x}$ and $\omega_{x_{\alpha}} \omega_{y}$ converges to $\omega_{x} \omega_{y}$. Fix $t \in S$. Then there exist $p, q \in S$ such that $t=p q$. Then $\omega_{x_{\alpha}}(t)=\omega_{x_{\alpha}}(p q)=$ $\omega_{x_{\alpha}} \omega_{p}(q)$ which converges to $\omega_{x} \omega_{p}(q)=\omega_{x}(p q)=\omega_{x}(t)$. Similarly, $t \omega_{x_{\alpha}}$ converges to $t \omega_{x}$. Thus $\omega_{x_{\alpha}}$ converges to $\omega_{x}$ in $\Omega_{p}(S)$.

If $S$ is a topological semigroup, we show $\pi(S)$ is a topological semigroup with the subspace topology from $\Omega_{p}(S)$. Let $\omega_{x_{\alpha}} \rightarrow \omega_{x}$ and $\omega_{y_{\beta}} \rightarrow \omega_{y}$. Let $s \in S$ and let $L$ be a compact left ideal containing $s$. Then for all $\alpha, \beta$ we have $\omega_{x_{\alpha}} \omega_{y_{\beta}} s=x_{\alpha} y_{\beta} s \in L$. Let $p$ be a cluster point of the product net $x_{\alpha} y_{\beta} s$. Then for all $q \in S q x_{\alpha} y_{\beta} s$ clusters to $q p$ and $q x_{\alpha} y_{\beta} s=\left(q \omega_{x_{\alpha}}\right)\left(\omega_{y_{\beta}} s\right)$ converges 
to $\left(q \omega_{x}\right)\left(\omega_{y} s\right)=q x y s$. Hence $q p=q x y s$ and by reductivity $p=x y s$. Since the only cluster point of the net $x_{\alpha} y_{\beta} s$ in the compact set $L$ is $x y s, \omega_{x_{\alpha}} \omega_{y_{\beta}} s$ converges to $\omega_{x} \omega_{y} s$. Using this fact and its right analogue, we have $\omega_{x_{\alpha}} \omega_{y_{\alpha}}$ converges to $\omega_{x} \omega_{y}$ in $\Omega_{p}(S)$.

By Propositions 3.2 and 1.4 (and 3.4), the natural mapping from $\pi(S)$ into its translational hull will be a topological embedding into a compact semitopological (or topological in the case that $S$ was a topological semigroup) semigroup. Let $T$ be the closure of the image of $\pi(S)$ in its translational hull. The composition $f$ of $\pi$ with the mapping from $\pi(S)$ into $T$ gives a monomorphic compactification $(T, f)$.

All that remains is to show that $T$ is weakly reductive. Suppose $\omega_{1}, \omega_{2}$ $\in T$ such that $\omega_{1} \omega=\omega_{2} \omega$ and $\omega \omega_{1}=\omega \omega_{2}$ for all $\omega \in T$. Let $x \in \pi(S)$. There exist $y, z \in \pi(S)$ such that $x=y z$. Then

$$
\omega_{1} x=\omega_{1}(y z)=\left(\omega_{1} \omega_{y}\right) z=\left(\omega_{2} \omega_{y}\right) z=\omega_{2}(y z)=\omega_{2} x
$$

similary, $x \omega_{1}=x \omega_{2}$ for any $x \in \pi(S)$. Hence $\omega_{1}=\omega_{2}$.

It is interesting to note that one of the ingredients in the proof of 3.5 is that if $S$ is a globally idempotent semigroup and $T$ is a subsemigroup of $\Omega(S)$ containing $\pi(S)$, then $T$ is weakly reductive.

Corollary 3.5 gives sufficient conditions for the existence of monomorphic compactifications. We now consider the problem of uniqueness of such compactifications.

An ideal $I$ in a semigroup $S$ is algebraically dense if the identity relation is the only congruence on $S$ which when restricted to $I$ is the identity relation on $I$. Generally $I$ is called simply a dense ideal, but we employ that terminology for topological denseness.

\subsection{Proposition. If I is a dense ideal of a weakly reductive semitopo-} logical semigroup $S$, then $I$ is algebraically dense.

Proof. Let $\rho$ be a congruence on $S$, and suppose there exists $(y, z) \in \rho$ such that $y \neq z$. Since $S$ is weakly reductive, there exists $x \in S$ such that $x y \neq$ $x z$ or $y x \neq z x$; assume the former. There exist open sets $U$ and $V$ such that $x y \in U, x z \in V$, and $U \cap V=\varnothing$. Choose an open set $W$ such that $x \in W, W y$ $\subset U$, and $W z \subset V$. Since $I$ is dense, there exists $b \in W \cap I$. Then $(b y, b z) \in \rho$ and $b y \neq b z$. Hence $I$ is algebraically dense.

3.7. Proposition. If I is an ideal of a semitopological semigroup $S$, then the mapping $\sigma: S \rightarrow \Omega_{p}(I)$ which sends $s$ to the inner bitranslation $\omega_{s}$ restricted to $I$ is a continuous homomorphism making the following diagram commute: 


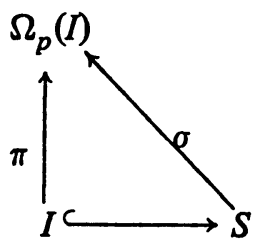

Proof. Since multiplication is separately continuous in $S, \sigma$ is continuous. It is well known from the algebraic theory that $\sigma$ is a homomorphism making the diagram commute $[10$, p. 296].

3.8. Proposition. If I is a dense ideal of a compact weakly reductive semitopological semigroup $S$, then $S$ is topologically isomorphic to the closure of $\pi(I)$ in $\Omega_{p}(I)$.

Proof. Consider the diagram

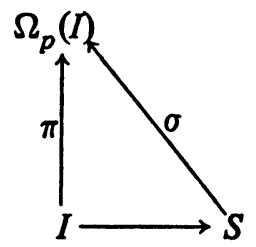

Proposition 1.4 and 1.8 imply that $\pi$ is a topological isomorphism into. Since the diagram commutes and by $3.6, I$ is algebraically dense, it must be the case that $\sigma$ is one-to-one. By 3.7, $\sigma$ is continuous; hence $\sigma$ is a topological isomorphism into. Now $\sigma(S)=\sigma\left(I^{*}\right)=\sigma\left(I^{*}=\pi(I)^{*}\right.$ and the proof is complete.

3.9. Proposition. If $S$ is a semitopological semigroup which is the union of compact right ideals and the union of compact left ideals, then $S$ has at most one weakly reductive monomorphic compactification.

We again have need of an additional lemma before we prove this proposition.

3.10. LEMmA. Let $S$ be a semitopological semigroup, $L$ a family of left ideals such that $S=\bigcup_{L}$ and $R$ a family of right ideals such that $S=U_{R}$. Let $j: S \rightarrow T$ be an algebraic isomorphism onto a weakly net reductive semitopological semigroup $T$ which when restricted to each $L \in L$ and $R \in R$ is continuous. Then $j$ is continuous.

Proof. Let $x_{\alpha} \rightarrow x$ in $S$. Since $T$ is weakly net reductive, it suffices to show $y j\left(x_{\alpha}\right) \rightarrow y j(x)$ and $j\left(x_{\alpha}\right) y \rightarrow j(x) y$ for each $y \in T$. For this purpose, let $y \in T$ and let $z \in S$ such that $j(z)=y$. Let $R \in R$ such that $z \in R$. Then $z x_{\alpha} \rightarrow z x$ in $S$ and hence in $R$. Since $j \mid R$ is continuous, $y j\left(x_{\alpha}\right)=j(z) j\left(x_{\alpha}\right)=$ $j\left(z x_{\alpha}\right) \rightarrow j(z x)=j(z) j(x)=y j(x)$. Similarly, $j\left(x_{\alpha}\right) y \rightarrow j(x) y$. 
Proof of Proposition 39. Let $\left(T_{1}, f_{1}\right)$ and $\left(T_{2}, f_{2}\right)$ be two monomorphic weakly reductive semigroup compactifications of $S$. Consider the diagram

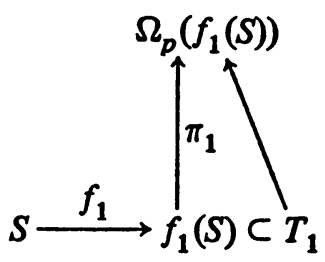

By 3.8 and 3.1, $T_{1}$ is topologically isomorphic to the closure of $\pi_{1} f_{1}(S)$ in $\Omega_{p}\left(f_{1}(S)\right)$. Similarly $T_{2}$ is topologically isomorphic to the closure of $\pi_{2} f_{2}(S)$ in $\Omega_{2}\left(f_{2}(S)\right)$.

Now let $j=f_{2} \circ f_{1}^{-1}: f_{1}(S) \rightarrow f_{2}(S)$. Then $j$ is an algebraic isomorphism which is continuous when restricted to $f_{1}(L)$ or $f_{1}(R)$ for any compact left ideal $L$ or compact right ideal $R$ of $S$. By $1.8, f_{2}(S)$ is weakly net reductive. Hence by Lemma $3.10, j$ is continuous. Similarly $j^{-1}$ is continuous. Thus $f_{1}(S)$ and $f_{2}(S)$ are topologically isomorphic. Hence $\Omega_{p}\left(f_{1}(S)\right)$ is topologically isomorphic to $\Omega_{p}\left(f_{2}(S)\right)$, and thus the closure of $\pi_{1} f_{1}(S)$ in $\Omega_{p}\left(f_{1}(S)\right)$ is topologically isomorphic to the closure of $\pi_{2} f_{2}(S)$ in $\Omega_{p}\left(f_{2}(S)\right)$.

As an immediate corollary of Propositions 3.5 and 3.9 we have

3.11. CoROllaRY. If $S$ is a reductive globally idempotent semitopological semigroup which is the union of compact left ideals and the union of compact right ideals, then $S$ has precisely one monomorphic weakly reductive compactification.

3.12. CoROllaRY. Let I be a dense ideal of a compact topological semigroup $S$ where $S=E S E$ ( $E$ denotes the set of idempotents). Then $S$ is the only topological semigroup which is a monomorphic compactification of $I$.

Proof. Since $S=E S E, S$ is weakly reductive (see e.g. $[6$, p. 89]). Let $(T, f)$ be a monomorphic compactification of $I$. Then $I \subset S=E S E$ implies $I=E I E$, so that $f(I)=f(E) f(I) f(E) \subset E f(I) E$, which implies $T=f(I)^{*} \subset$ $(E f(I) E)^{*} \subset E f(I)^{*} E=E T E$. Hence $T=E T E, T$ is weakly reductive (as above), and the result follows from 3.9 .

3.13. COROLLARY. Let I be a dense ideal of a compact semitopological semigroup $S$ where $S$ has a right (or left) identity. Then $S$ is the only monomorphic compactification of $I$.

Proof. It is straightforward to verify that any compactification of $I$ must have a right identity, and hence be weakly reductive. The corollary then follows from Proposition 3.9. 
3.14. Corollary. Let I be a dense ideal of a compact reductive semitopological semigroup $S$. If multiplication restricted to $I \times I$ is jointly continuous, then $S$ is a topological semigroup.

Proof. By $1.8, I$ is net reductive. Hence by 1.7 and 1.1 , multiplication in $\Omega_{p}(I)$ is jointly continuous. It follows from 3.8 that multiplication in $S$ is jointly continuous.

We close this section with some isomorphism theorems.

3.15. Proposition. Let I be a dense ideal in a compact reductive semitopological semigroup $S$ such that $S I=I=I S$. Then $\Omega_{p}(S)$ is topologically isomorphic to $\Omega_{p}(I)$.

Proof. By 2.4 we have the following commutative diagram

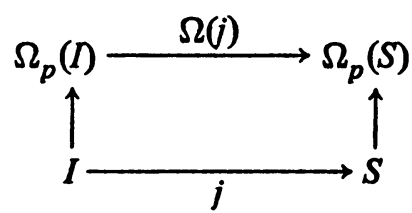

where $j: I \rightarrow S$ is the inclusion mapping. For $\omega \in \Omega_{p}(S)$, we show that $\omega I \cup$ $I \omega \subset I$. Let $x \in I$. Then there exist $y \in S, z \in I$ such that $x=y z$. We have $\omega x=\omega(y z)=(\omega y) z \in I$. Similarly $x \omega \in I$.

Hence there exists a mapping $r: \Omega_{p}(S) \rightarrow \Omega_{p}(I)$ which restricts a bitranslation to $I$ (that $r$ is continuous is immediate from the definition of the topology of pointwise convergence). Since $\Omega(j)$ is the unique extension of a bitranslation to $S$ and $r$ is the restriction, the two homomorphisms are inverses for each other, and hence each is a topological isomorphism.

3.16. COROLlary. Let I be a dense ideal of a compact semitopological monoid $S$. Then $S$ is topologically isomorphic to $\Omega_{p}(I)$.

Proof. By $3.15, \Omega_{p}(S) \cong \Omega_{p}(I)$. But since $S$ has an identity $\Omega_{p}(S) \cong S$.

Many of the results of this section had been derived previously by other techniques. The translational hull provides a unifying and more natural approach to these results.

Corollary 3.5 is a generalization of Theorem 2 of [5] to the nonabelian case.

Analogues of 3.9, 3.11, 3.12, and 3.13 are found in [5]. The interested reader may refer to that paper for other similar corollaries which we could have also derived in this context. Corollary 3.14 appears as Proposition 7.5 in [9].

4. An application to divisibility. A semigroup $S$ is said to be power-cancellative if $a, b \in S$ and $a^{n}=b^{n}$ for some positive integer $n$ implies that $a=b$. 
Observe that a power-cancellative abelian semigroup $S$ is reductive. To see this, let $a, b \in S$ and suppose that $t a=t b$ for all $t \in S$. Then for $t=a$ we have. $a^{2}=a b$ and for $t=b$ we have $b a=b^{2}$, and so $a^{2}=a b=b a=b^{2}$. Since $S$ is power-cancellative, we obtain that $a=b$, and hence $S$ is reductive.

4.1. Lemma. Let $S$ be a power-cancellative abelian semigroup. Then $\Omega(S)$ is a power-cancellative abelian semigroup.

Proof. Since $S$ is power-cancellative, we have that $S$ is reductive, and hence $\Omega(S)$ is abelian (see [11]). To see that $\Omega(S)$ is power-cancellative, let $\omega_{1}, \omega_{2} \in \Omega(S)$ and suppose that $\omega_{1}^{n}=\omega_{2}^{n}$ for some positive integer $n$. Using induction and the fact that $\omega x=x \omega$ for each $\omega \in \Omega(S)$ and $x \in S$, we obtain that $(\omega x)^{n}=\omega^{n} x^{n}$ for each $x \in S$. Let $x \in S$. Then $\left(\omega_{1} x\right)^{n}=\omega_{1}^{n} x^{n}=\omega_{2}^{n} x^{n}$ $=\left(\omega_{2} x\right)^{n}$, and since $S$ is power-cancellative, we have that $\omega_{1} x=\omega_{2} x$. It follows that $\omega_{1}=\omega_{2}$ and $\Omega(S)$ is power-cancellative.

4.2. Lemma. Let $S$ be a topological semigroup, I a closed ideal of $S$, and $\Gamma=\left\{\omega \in \Omega_{p}(S): \omega I \cup I \omega \subset I\right\}$. Then $\Gamma^{\sim}$ is a closed subsemigroup of $\Omega_{p}(S)$ containing $\pi(S)$.

Proof. That $\pi(S) \subset \Gamma^{\sim}$ and that $\Gamma^{\sim}$ is a subsemigroup of $\Omega_{p}(S)$ are transparent. To see that $\Gamma$ is closed, let $\omega_{\alpha} \rightarrow \omega$. Then for each $x \in I$, we have $\omega_{\alpha} x \rightarrow \omega x, \omega_{\alpha} x \in I$ for each $\alpha$, and since $I$ is closed, $\omega x \in I$, and similarly $x \omega \in I$. It follows that $\omega \in \Gamma^{\sim}$ and $\Gamma^{\sim}$ is closed.

A semigroup $S$ is said to be [uniquely] divisible if for each $x \in S$ and each positive integer $n$, there exists a [unique] element $y \in S$ such that $y^{n}=x$. Ob. serve that a divisible subsemigroup of a power-cancellative semigroup is uniquely divisible.

\subsection{THEOREM. Let $S$ be a compact power-cancellative abelian topological} semigroup such that $S_{n}=\left\{x^{n}: x \in S\right\}$ is an ideal of $S$ for each positive integer $n$. Then there exists a compact uniquely divisible abelian subsemigroup $T$ of the compact topological semigroup $\Omega_{p}(S)$ such that $\pi$ is an embedding of $S$ into $T$, where $\pi: S \rightarrow \Omega_{p}(S)$ is the canonical embedding.

Proof. Let $N$ denote the set of positive integers and let $T=\bigcap\left\{S_{n}^{\sim}: n \in N\right\}$. In view of 4.2 and 1.11 , we see that $T$ is a compact subsemigroup of $\Omega_{p}(S)$ containing $\pi(S)$, and $S$ is topologically isomorphic to $\pi(S)$. To complete the proof, we will show that $T$ is uniquely divisible. Notice that it is sufficient to show that $T$ is divisible, since $\Omega_{p}(S)$ is, by virtue of 4.1 , power-cancellative.

Let $\omega \in T$ and $n \in N$. Now for each $x \in S$, we have that $\omega x^{n} \in S_{n}$, since $\omega \in S_{n}^{\sim}$, so that there exists a unique (since $S$ is power-cancellative) $x_{0} \in S$ such that $x_{0}^{n}=\omega x^{n}$. Define $\tau x=x_{0}$ (and $x \tau=x_{0}$ ) for each $x \in S$. 
To demonstrate that $\tau$ is a bitranslation of $S$, we need only show that $\tau$ has the linking property (see 1.9). For this purpose, let $a, b \in S$. Then $[a(\tau b)]^{n}$ $=a^{n}(\tau b)^{n}=a^{n} b_{0}^{n}=a^{n}\left(\omega b^{n}\right)=\left(a^{n} \omega\right) b^{n}=a_{0}^{n} b^{n}=\left(a_{0} b\right)^{n}=[(a \tau) b]^{n}$. Since $S$ is power-cancellative, we have that $a(\tau b)=(a \tau) b$, and $\tau$ is a bitranslation of $S$. From 1.11, we see that $\tau \in \Omega_{p}(S)$.

For the purpose of showing that $\tau^{n}=\omega$, let $x \in S$ and observe that $\left[\tau^{n} x\right]^{n}$ $=(\omega x)^{n}$ by employing induction and the definition of $\tau$. Again, since $S$ is powercancellative, we have $\tau^{n} x=\omega x$, and hence $\tau^{n}=\omega$.

Finally, to see that $\tau \in T$, let $k \in N$ and $x \in S$. We will show that $\tau x^{k} \in$ $S_{k}$. Now $\left[\tau x^{k}\right]^{n}=\left(x^{k}\right)_{0}^{n}=\omega x^{k n}=u^{k n}$ for some $u \in S$, since $\omega \in T \subset S_{k n}^{\sim}$. We obtain that $\left[\tau x^{k}\right]^{n}=\left[u^{k}\right]^{n}$, and by power-cancellativity, we have $\tau x^{k}=$ $u^{k} \in S_{k}$.

5. Computational techniques and examples. In this section we present numerous examples of translational hulls along with some results which give a method of performing this computation for certain classes of semigroups.

Although our results do not rely on the minimality condition of this notion, it is sometimes convenient to locate a minimal subset of a semigroup $S$ which determines the bitranslations of $S$. This leads to the concept of a basis.

If $S$ is a semitopological semigroup, then a subset $B$ of $S$ is called a bitranslational determination subset of $S$ (or simply a determination subset of $S$ ) provided that whenever $\omega$ and $\omega^{\prime}$ are continuous bitranslations of $S$ such that $\omega b=$ $\omega^{\prime} b$ and $b \omega=b \omega^{\prime}$ for each $b \in B$, then $\omega=\omega^{\prime}$. A minimal determination subset of $S$ is called a bitranslational basis for $S$ (or simply a basis for $S$ ).

A trivial example of a basis is $\{1\}$ in a semitopological semigroup $S$ with identity 1 .

If $S$ is a semitopological semigroup containing a cancellative element $x$ such that $S x=x S$, then $\{x\}$ is a basis for $S$. To see this, suppose that $\omega$ and $\omega^{\prime}$ are bitranslations of $S$ such that $\omega x=\omega^{\prime} x$ and $x \omega=x \omega^{\prime}$, and let $a \in S$. Then, since $S x=x S$, we have that $a x=x c$ for some $c \in S$, and $(\omega a) x=\omega(a x)=$ $\omega(x c)=(\omega x) c=\left(\omega^{\prime} x\right) c=\omega^{\prime}(x c)=\omega^{\prime}(a x)=\left(\omega^{\prime} a\right) x$. Since $x$ is cancellative, we have $\omega a=\omega^{\prime} a$, and similarly $a \omega=a \omega^{\prime}$. It follows that $\omega=\omega^{\prime}$ and $\{x\}$ is a basis for $S$.

Observe that for any semitopological semigroup $S$ that $S$ itself is a determination subset of $S$. We defer our discussion on the existence of a basis to the end of this section, and introduce at this point an important related concept.

If $S$ is a semigroup and $B$ is a subset of $S$, then $B$ is called a separating subset of $S$ if for each distinct pair $x$ and $y$ of elements of $S$, there exist $a, b \in B$ such that $a x \neq a y$ and $x b \neq y b$.

Note that a semigroup with a separating subset is reductive. In this context we have the following: 
5.1. Proposition. Let $S$ be a reductive abelian semitopological semigroup and let $B$ be a subset of $S$. Then $B$ is a separating subset of $S$ if and only if $B$ is a determination subset of $S$. In particular, $B$ is a minimal separating subset of $S$ if and only if $B$ is a basis for $S$.

Proof. Suppose that $B$ is a determination subset of $S$, and let $x \neq y$ in S. Now if $a x=a y$ for all $a \in B$, then we would have $a \omega_{x}=a \omega_{y}$ for all $a \in B$, so that $\omega_{x}=\omega_{y}$ and hence $x=y$, since $S$ is reductive. It follows that $b x \neq b y$ for some $b \in B$, and that $B$ is a separating subset of $S$.

To establish the converse, suppose that $B$ is a separating subset of $S$, and let $\omega$ and $\omega^{\prime}$ be bitranslations of $S$ such that $\omega b=\omega^{\prime} b$ for each $b \in S$. Assume (for the purpose of contradiction) that $\omega x \neq \omega^{\prime} x$ for some $x \in S$. Then $(\omega x) u$ $\neq\left(\omega^{\prime} x\right) u$ for some $u \in B$, since $B$ is a separating subset of $S$. On the other hand, we have $(\omega x) u=\omega(x u)=\omega(u x)=(\omega u) x=\left(\omega^{\prime} u\right) x=\omega^{\prime}(u x)=\omega^{\prime}(x u)=$ $\left(\omega^{\prime} x\right) u$. This contradiction yields that $\omega x=\omega^{\prime} x$ for all $x \in S, \omega=\omega^{\prime}$, and $B$ is a determination subset of $S$.

Observe that a separating subset of a semitopological semigroup is always a determination subset without any additional hypothesis on the semigroup.

Proposition 5.1 will be employed in a later result in this section. We begin now our list of examples with the simplest kind of example.

5.2. EXAMPLE. Let $S$ be a Hausdorff space with zero multiplication, i.e., pick $0 \in S$ and define $x y=0$ for all $x, y \in S$. Then $S$ is a topological semigroup, and the left (and right) translations of $S$ are precisely those functions $f: S \rightarrow S$ with $f(0)=0$ and each left translation is linked with each right translation. It follows that $\Lambda(S)=P(S)=\left\{f \in S^{S}: f(0)=0\right\}$ and $\Omega(S)=\Lambda(S) \times$ $P(S)$. Thus, in particular, if $S=[0,1]$ with the usual topology and zero multiplication, then $\Omega_{p}(S)$ is not compact and $S$ does not have a basis.

To obtain a compact right reductive topological semigroup $S$ such that $\Omega_{p}(S)$ is not compact consider the following:

5.3. EXAMPLE. Let $S$ be any Hausdorff space with left trivial multiplication, i.e., $x y=x$ for all $x, y \in S$. Then $S$ is a topological semigroup which is right reductive. Let $\lambda: S \rightarrow S$ be any function, and let $x, y \in S$. Then we have $\lambda(x y)=\lambda x=(\lambda x) y$, so that $\lambda$ is a left translation of $S$. Suppose that $\rho$ is a right translation of $S$. Then, for $x, y \in S$, we have $x \rho=(x y) \rho=x(y \rho)=x$, and hence $\rho=1_{S}$ (the identity translation). We obtain that $\Lambda(S)=S^{S}, P(S)=$ $\left\{1_{S}\right\}$, and $\Omega(S)=\Lambda(S) \times P(S)=\left\{\left(\lambda, 1_{S}\right): \lambda \in S^{S}\right\}$. In particular, if $S=[0,1]$ with left trivial multiplication, then $\Omega_{p}(S)$ is homeomorphic to $S^{S}$ with the topology of pointwise convergence, and hence $\Omega_{p}(S)$ is not compact. Moreover, $S$ has no basis.

There is an obvious dual example to 5.3.

To establish our first computational algorithm (5.5), we will employ the following: 
5.4. Proposition. Let $S$ be a compact semitopological semigroup with identity 1 , and let $x$ be a right cancellative element of $S$ such that $S x \subset x S$. Then $\Lambda_{p}(S x)$ is topologically isomorphic to $S$.

Proof. Define $\theta: S \rightarrow \Lambda_{p}(S x)$ by $\theta(a)=\lambda_{a} \mid S x$. Then $\theta$ is clearly a continuous homomorphism.

Suppose that $a, b \in S$ and $\theta(a)=\theta(b)$. Then $a x=\lambda_{a} x=\lambda_{b} x=b x$, and $a=b$, since $x$ is right cancellative. It follows that $\theta$ is injective.

For the purpose of showing that $\theta$ is surjective, let $\lambda \in \Lambda_{p}(S x)$ and let $a \in$ $S$ such that $\lambda x=a x$. We will demonstrate that $\theta(a)=\lambda$. Let $y \in S x$. Then $y$ $=t x=x t^{\prime}$ for some $t, t^{\prime} \in S$, since $S x \subset x S$. We obtain that $(\lambda y) x=\lambda(y x)=$ $\lambda\left(x t^{\prime} x\right)=(\lambda x) t^{\prime} x=(a x)\left(t^{\prime} x\right)=a\left(x t^{\prime}\right) x=a y x=\left(\lambda_{a} y\right) x$, so that $\lambda y=\lambda_{a} y$, since $x$ is right cancellative. It follows that $\lambda=\lambda_{a} \mid S x=\theta(a)$, and $\theta$ is surjective.

The conclusion now follows from the fact that $S$ is compact and $\Lambda_{p}(S x)$ is Hausdorff.

There is an obvious dual to 5.4. We also remark that the proof of the algebraic analogue of 5.4 is contained in the proof of 5.4. The same is true of the following:

5.5. THEOREM. Let $S$ be a compact semitopological semigroup with 1 and let $x$ be a cancellative element of $S$ such that $S x=x S$. Then $\Omega_{p}(S x)$ is topologically isomorphic to $S$.

Proof. Let $\theta: S \rightarrow \Lambda_{p}(S x)$ and $\gamma: S \rightarrow P_{p}(S x)$ be the topological isomorphism of 5.4 and its dual, respectively, and let $\pi_{1}: \Omega_{p}(S x) \rightarrow \Lambda_{p}(S x)$ and $\pi_{2}: \Omega_{p}(S x) \rightarrow P_{p}(S x)$ be projections. Define $\varphi: S \rightarrow \Omega_{p}(S x)$ by $\varphi(a)=\omega_{a} \mid S x$. Then $\varphi$ is a continuous homomorphism and the diagram:

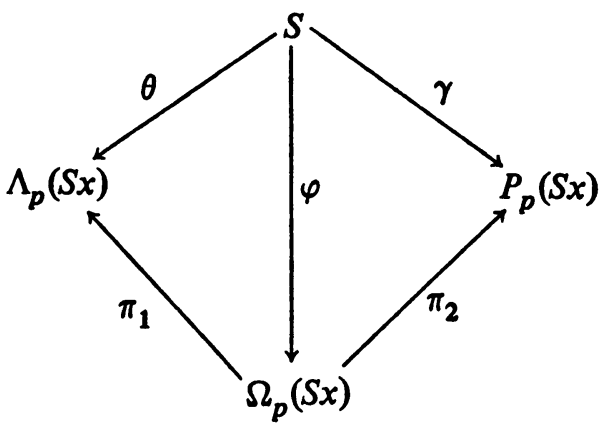

commutes. It is clear from this diagram that $\varphi$ is injective.

To see that $\varphi$ is surjective, let $\omega=(\lambda, \rho) \in \Omega_{p}(S x)$. Then $\lambda=\pi_{1} \omega=$ $\lambda_{a} \mid S x$ for some $a \in S$, since $\theta$ is an isomorphism, and similarly $\rho=\pi_{2} \omega=\rho_{b} \mid S x$ for some $b \in S$. We have that $x a x=x(a x)=x(\lambda x)=(x \rho) x=(x b) x=x b x$, and $a=b$, since $x$ is cancellative. It follows that $\omega=\omega_{a} \mid S x=\varphi(a), \varphi$ is surjective, and hence $\varphi$ is a topological isomorphism. 
Observe in 5.5 that if $S$ is a topological semigroup, then $S x$ is a compact reductive topological semigroup, so that $\Omega_{p}(S x)=\Omega_{c}(S x)$ by virtue of 1.11 . However, we hasten to point out that the joint continuity of the multiplication in $S$ would yield the continuity of the map $\varphi$ in the diagram in the proof of 5.5 if " $p$ " were replaced by " $c$ "; thus providing an alternative proof of this fact.

Our next three examples are applications of 5.5.

5.6. ExAMPLE. Let $S=[0,1]$ with the usual multiplication and topology and let $x=1 / 2$. Then $S x=[0,1 / 2]$, and by 5.5 , we have that $\Omega_{p}(S x)$ is topologically isomorphic to $S$.

5.7. ExAmple. Let $S=[0,1 / 2] \cup\{1\}$ with the usual multiplication and topology and let $x=1 / 2$. Then $S x=[0,1 / 4] \cup\{1 / 2\}$ and $\Omega_{p}(S x)$ is topologically isomorphic to $S$.

5.8. ExAmple. Let $R$ denote the real line, $T=\{(x, y) \in R \times R: 0 \leqslant x$ $\leqslant 1$ and $1 \leqslant x+y$, with multiplication $(x, y)(c, d)=(x c, x d+y)$, and let $S$ be the one-point compactification of $T$ with the ideal point acting as a zero for $S$. Then the element $a=(1 / 2,1 / 2)$ is cancellative and $S a=a S$, so that $\Omega_{p}(S a)$ is topologically isomorphic to $S$. The semigroup $T$ and $T a$ are pictured in the following illustration:

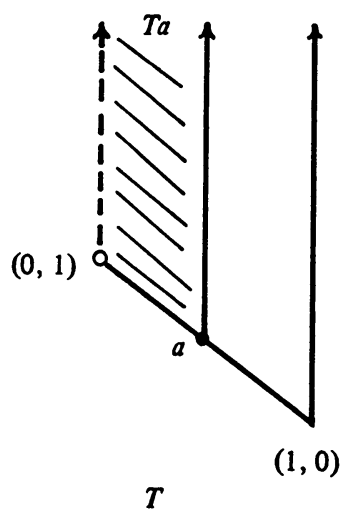

We turn now to our second computational algorithm. The following notation will be used: $N$ denotes the set of all positive integers, and for a subset $A$ of a semigroup $S$, let $D(A)=\left\{x \in S: x^{n} \in A\right.$ for some $\left.n \in N\right\}$.

5.9. THEOREM. Let $S$ be a compact uniquely divisible abelian topological semigroup and let $I$ be an ideal of $S$ such that $D(I)$ is dense in $S$. Then $\Omega_{p}(I)$ is topologically isomorphic to $\Omega_{p}(S)$.

Proof. Note that $S$ is power-cancellative and hence reductive and that $S=E S[6,3.1]$. It follows that $D(I)=E D(I)$ and from this that $D(I)=S D(I)$. Since $D(I)$ is a dense ideal of $S$, we have from 3.15 (and its proof) that $\Omega(j)$ : 
$\Omega_{p}(D(I)) \rightarrow \Omega_{p}(S)$ is a topological isomorphism, where $j: D(I) \rightarrow S$ is inclusion. Let $\psi=\Omega(j)$ and note from 2.4 that the diagram:

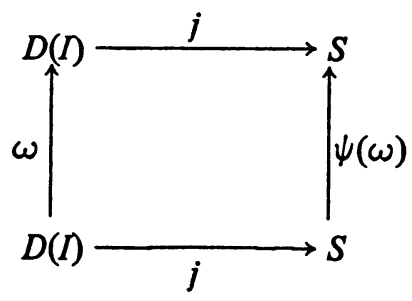

commutes for each $\omega \in \Omega_{p}(D(I))$.

Since $S=E S[6,3.1]$, we have that for each $\omega \in \Omega_{p}(S), \omega \mid I$ is a bitranslation of $I$. For if $x \in I$, then there exists $e \in E$ with $e x=x$, so that $\omega x=$ $\omega(e x)=(\omega e) x \in I$. Thus there is a continuous homomorphism $\varphi: \Omega_{p}(S) \rightarrow$ $\Omega_{p}(I)$ given by $\varphi(\omega)=\omega \mid I$. Note that $\varphi$ is injective, since if $\omega, \omega^{\prime} \in \Omega_{p}(S)$, and $\omega$ and $\omega^{\prime}$ agree on $I$, then they must agree on $D(I)$ and hence on $S$.

To see that $\varphi$ is surjective, it suffices to show that $\varphi \circ \psi$ is surjective. Note that for each $\omega \in \Omega_{p}(D(I))$, we have that $\varphi \circ \psi(\omega)=\omega \mid I$ by commutativity of the diagram above. Thus, it is sufficient to show that each bitranslation of $I$ can be extended to a continuous bitranslation of $D(I)$.

Let $\omega \in \Omega_{p}(I)$. For $x \in D(I)$, define $x \omega^{\prime}=\omega^{\prime} x=\left(\omega^{n} x^{n}\right)^{1 / n}$, where $x^{n}$ $\in I$. To see that $\omega^{\prime}$ is well defined, suppose that $m$ and $n$ are positive integers with $x^{m}, x^{n} \in I$. We obtain that $\left(\omega^{n} x^{n}\right)^{m}=\omega^{n m} x^{n m}=\left(\omega^{m} x^{m}\right)^{n}$, so $\left(\omega^{n} x^{n}\right)^{1 / n}=\left(\omega^{m} x^{m}\right)^{1 / m}$. Thus $\omega^{\prime}$ is well defined. To see that $\omega^{\prime}$ is a bitranslation of $I$, it is sufficient to demonstrate that $\omega^{\prime}$ has the linking property. For $x, y \in D(I)$, with $x^{n}, y^{m} \in I$, we have $\left[\left(x \omega^{\prime}\right) y\right]^{m n}=\left(x \omega^{\prime}\right)^{m n} y^{m n}=$ $\left(x^{m n} \omega^{m n}\right) y^{m n}=x^{m n}\left(\omega^{m n} y^{m n}\right)=x^{m n}\left(\omega^{\prime} y\right)^{m n}=\left[x\left(\omega^{\prime} y\right)\right]^{m n}$. Thus $\left(x \omega^{\prime}\right) y$ $=x\left(\omega^{\prime} y\right)$ and $\omega^{\prime}$ is a bitranslation extending $\omega$. Since $\omega^{\prime}$ is continuous by 2.3 , we have that $\omega^{\prime} \in \Omega_{p}(D(I))$ and $\varphi \circ \psi\left(\omega^{\prime}\right)=\omega$, so $\varphi$ is onto. Since $\Omega_{p}(S)$ is compact by 1.11 and $\Omega_{p}(I)$ is Hausdorff, we conclude that $\varphi$ is a topological isomorphism.

As an application of 5.9, we have the following:

5.10. ExAMPLE. Let $[0,1]$ denote the usual multiplicative real interval, and let $S=[0,1] \times[0,1]$. Let $I=\{(x, y) \in S: x+y \leqslant 1\}$. Then by 5.9, we have that $\Omega_{p}(I)$ is topologically isomorphic to $S$. In fact, any ideal of $S$ containing a neighborhood of $(0,0)$ has $S$ as its translational hull.

We proceed to our third computational algorithm.

5.11. THEOREM. Let $S$ be a compact reductive abelian semitopological semigroup and let $B$ be a separating subset of $S$ consisting of idempotents. Define $\varphi: \Omega_{p}(S) \rightarrow \bar{\Pi}\{S e\}_{e \in B}$ so that $\pi_{e} \varphi(\omega)=\omega e$, where $\pi_{e}$ is projection onto Se. Then $\varphi$ is an embedding. 
Proof. Now $\Omega_{p}(S)$ is a compact abelian semitopological semigroup and $x \omega=\omega x$ for each $\omega \in \Omega_{p}(S)$ and each $x \in S$. Clearly $\varphi$ is continuous, since its composition with each projection is continuous, and the injectivity of $\varphi$ follows from 5.1. To complete the proof we need to show that $\varphi$ is a homomorphism. For this purpose, let $\omega, \omega^{\prime} \in \Omega_{p}(S)$ and $e \in B$. Then $\pi_{e} \varphi\left(\omega \omega^{\prime}\right)=\omega \omega^{\prime} e=$ $\omega\left(e \omega^{\prime}\right)=\omega\left(e e \omega^{\prime}\right)=\omega\left(e\left(e \omega^{\prime}\right)\right)=(\omega e)\left(e \omega^{\prime}\right)=(\omega e)\left(\omega^{\prime} e\right)=\pi_{e} \varphi(\omega) \pi_{e} \varphi\left(\omega^{\prime}\right)$, and hence $\varphi$ is a homomorphism.

As an application of 5.11, we have the following:

5.12. ExAMPLE. Let $I=[0,1]$ with the usual topology and addition defined by $a+b=\min \{1, a \oplus b\}$, where $a \oplus b$ is the usual sum of $a$ and $b$, i.e., $I$ is the additive nilpotent interval. Let $P=I \times I$ and $S=\{(x, 1): x \in I\}$ $\{(1, y): y \in I \cup\{(x, 1-x): x \in I\}$. Then $S$ is a compact abelian reductive subsemigroup of $P$. Observe that $S$ is a semigroup on the 1-sphere. It is pictured in the following illustration:

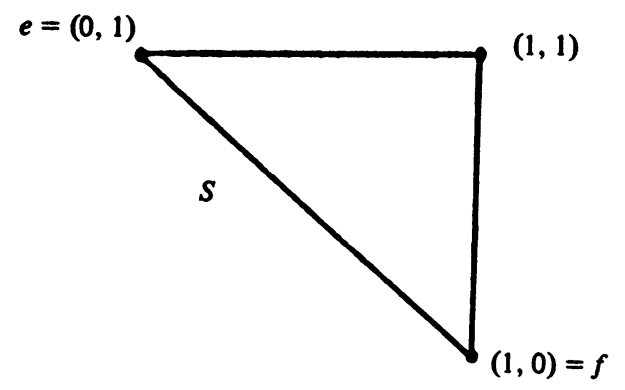

Observe that $e=(0,1)$ and $f=(1,0)$ is a minimal separating subset of $S$, since addition by $e$ and $f$ are projections. It follows from 5.11 , that $\omega \mapsto(\omega e, \omega f)$ is an embedding of $\Omega_{p}(S)$ into $S e \times S f=P$, so that $\Omega_{p}(S)$ is a subsemigroup of $P$ under this identification. We will show that $\Omega_{p}(S)=\{(a, b) \in P: a+b \geqslant 1\}$ $U\{(0,0)\}$, which is illustrated below:

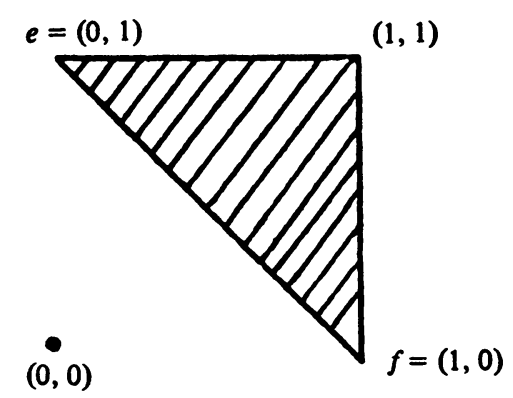

To verify this we show that only these elements of $P$ send $S$ into itself.

Let $(a, b) \in P$ with $a+b<1$ and suppose that $(a, b)+S \subseteq S$. Let $c=$ $(b+1-a) / 2$. Then $1-c=(a+1-b) / 2$ and $(c, 1-c) \in S$. We obtain that $(a, b)+(c, 1-c)=((a+b+1) / 2,(a+b+1) / 2)$, so that either $a+b$ 
$+1=2$ or $a+b+1=1$. The first condition is not possible, so that $a+b=$ 0 and $a=b=0$.

Suppose that $(a, b) \in P$ and $1 \leqslant a+b$. Then for $0 \leqslant x \leqslant 1,(a, b)+$ $(x, 1-x)=(a+x, b+1-x)$. Suppose that $a+x<1$. Then $1 \leqslant a+b<$ $b+1-x$, and so in any case $(a, b)+(x, 1-x) \in S$. Clearly, $(a, b)+(x, 1)$ and $(a, b)+(1, x)$ are in $S$, and the desired conclusion is obtained.

In our next algorithm we will modify the hypothesis of 5.12 and obtain a useful characterization of the translational hull of such a semigroup.

\subsection{THEOREM. Let $S$ be a compact abelian semitopological semigroup} and let $B$ be a separating subset of $S$ consisting of idempotents such that $S=$ $\bigcup\{S e\}_{e \in B}$. Let $K=\left\{x \in \Pi\{S e\}_{e \in B}: \pi_{e}(x) f=\pi_{f}(x) e\right.$ for each $\left.e, f \in B\right\}$. Then $K$ is a compact semitopological subsemigroup of $\Pi\{S e\}_{e \in B}$ and $K$ is topologically isomorphic to $\Omega_{p}(S)$.

Proof. Observe that $S=E S$, so that $S$ is reductive. A straightforward argument yields that $K$ is a closed subsemigroup of $\Pi\{S e\}_{e \in B}$ and hence is compact. We will demonstrate that $K$ is the image of $\Omega_{p}(S)$ under the embedding $\varphi$ of 5.11 .

To see that $K \subset \varphi\left(\Omega_{p}(S)\right)$, let $x \in K$. We will define a bitranslation $\omega$ of $S$ such that $\varphi(\omega)=x$. Let $t \in S$, with $t \in S e$ and define $\omega t=t \omega=\pi_{e}(x) t$. To show that $\omega$ is well defined, suppose also that $t \in S f$. Then $\pi_{e}(x) t=\pi_{e}(x)(t f)$ $=\pi_{e}(x) f t=\pi_{f}(x) e t=\pi_{f}(x) t$, and $\omega$ is well defined. To see that $\omega$ is a bitranslation of $S$, we need only demonstrate the linking property, since $S$ is reductive. For this purpose, let $a, b \in S$, with $a \in S u$ and $b \in S v$ for $u, v \in B$. Then $(a \omega) b$ $=\left[\pi_{u}(x) a\right] b=\left[\pi_{u}(x) b\right] a=\left(\pi_{u}(x) v b\right) a=\left(\pi_{v}(x) u b\right) a=\left(\pi_{v}(x) b\right)(u a)=(\omega b) a=$ $a(\omega b)$, and hence $\omega$ is a bitranslation of $S$. In view of the reductivity of $S$ and 1.11 , we see that $\omega \in \Omega_{p}(S)$. Now let $g \in B$. Then $\pi_{g} \varphi(\omega)=\omega g=\pi_{g}(x) g=$ $\pi_{g}(x)$, and hence $\varphi(\omega)=x$. We obtain that $K \subset \varphi\left(\Omega_{p}(S)\right)$.

To see that $\varphi\left(\Omega_{p}(S)\right) \subset K$, let $\omega \in \Omega_{p}(S)$, and let $e, f \in B$. Then $\pi_{e}(\varphi(\omega)) f=(\omega e) f=\omega(e f)=\omega(f e)=(\omega f) e=\pi_{f}(\varphi(\omega))$. It follows that $\varphi(\omega) \in K$ and $\varphi\left(\Omega_{p}(S)\right) \subset K$.

If $S$ is as in 5.13 and $B=\{e, f\}$, then $\Omega_{p}(S)$ defines a pull-back for the continuous homomorphisms $\theta_{1}:$ Se $\rightarrow$ Sef with $\theta_{1}(x e)=x e f$, and $\theta_{2}: S f \rightarrow$ Sef with $\theta_{2}(x f)=x e f$. To see this, define $\sigma_{1}: \Omega_{p}(S) \rightarrow$ Se by $\sigma_{1}(\omega)=\omega e$, and $\sigma_{2}: \Omega_{p}(S) \rightarrow S f$ by $\sigma_{2}(\omega)=\omega f$. Then the diagram:

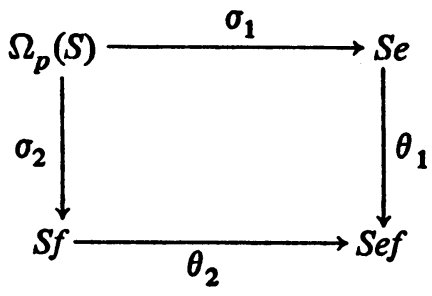


commutes. Suppose that $T$ is a compact abelian topological semigroup with 1 , and $\gamma_{1}: T \rightarrow S e$ and $\gamma_{2}: T \rightarrow S f$ such that $\theta_{1} \gamma_{1}=\theta_{2} \gamma_{2}$. Define $\gamma: T \rightarrow$ $\Omega_{p}(S)$ by $\gamma(t) x=\gamma_{1}(t) x$ if $x \in S e$, and $\gamma(t) x=\gamma_{2}(t) x$ if $x \in S f$. Then $\gamma$ is the morphism required to show that the above is a pull-back diagram.

As an application of 5.13, we construct a compact connected semilattice whose translational hull is not connected.

5.14. ExAMPLE. Let $M=[0,1]$ with the usual topology and min multiplication, i.e., $x y=\min \{x, y\}$, and let $P=M \times M$. Let $S=\{(0, b): b \in M\} \cup$ $\{(a, 0): a \in M\} \cup\{(a, 1): a \leqslant 1 / 2\} \cup\{(1, b): b \leqslant 1 / 2\} \cup\{(a, b) \in P: a \leqslant 1 / 2$, $b \leqslant 1 / 2\}$, and let $e=(1 / 2,1)$ and $f=(1,1 / 2)$. Then $S$ is a compact connected subsemilattice of $P$ with $\{e, f\}$ as a basis and $S=S e \cup S f$. We visualize $S$ in the following illustration:

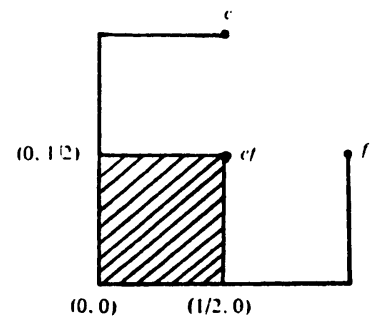

Let $S^{1}=S \cup\{(1,1)\}$ and let $K=\{(a, b) \in S e \times S f: a f=b e\}$. Then according to $5.13, \Omega_{p}(S)$ is topologically isomorphic to $K$. Define $\psi: S^{1} \rightarrow K$ by $\psi(t)=$ (te, $t f$ ) for each $t \in S^{1}$. A straightforward argument yields that $\psi$ is a topological isomorphism, and we conclude that $\Omega_{p}(S)$ is topologically isomorphic to $S^{1}$.

As a second application of 5.13, we present a more complicated version of the example of 5.14 which is a compact connected semilattice whose translational hull contains a denumerable set of isolated points.

5.15. ExAmple. Let $P$ and $S$ be as in 5.14, and let $N$ denote the set of all positive integers. For each $n \in N$, let $A_{n}=\left\{(a, b) \in P: a=1 / 2+1 / 2^{n}\right.$ and $0 \leqslant b \leqslant 1 / 2\}$ and let $B_{n}=\left\{(a, b) \in P: 0 \leqslant a \leqslant 1 / 2\right.$ and $\left.b=1 / 2+1 / 2^{n}\right\}$. Let $T=S \cup \cup\left\{A_{n}\right\}_{n \in N} \cup \bigcup\left\{B_{n}\right\}_{n \in N}$. Then $T$ is a compact connected subsemilattice of $P$ such that $T=T e \cup T f$, and is illustrated as follows:

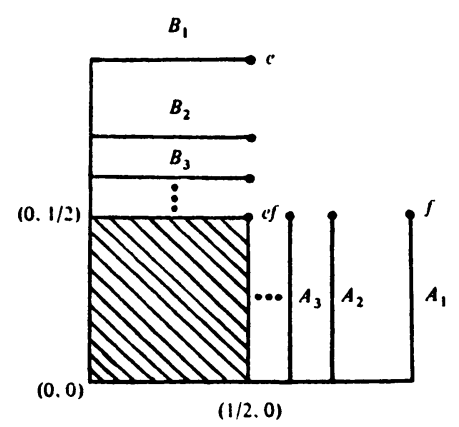


Observe, as in 5.14, that $\{e, f\}$ is a basis for $T$ and $T=T e \cup T f$. Let $T_{0}=T$ $\cup\left\{\left(1 / 2+1 / 2^{n}, 1 / 2+1 / 2^{m}\right): m, n \in N\right\}$, and let $K=\{(a, b) \in T e \times T f: a f=$ be\}. Then $T_{0}$ is a compact subsemilattice of $P$, and according to $5.13, \Omega_{p}(T)$ is topologically isomorphic to $K$. As in 5.14, define $\psi: T_{0} \rightarrow K$ by $\psi(t)=$ $(t e, t f)$ for each $t \in T_{0}$. Although somewhat tedious, it is straightforward to verify that $\psi$ is a topological isomorphism, and we conclude that $\Omega_{p}(T)$ is topologically isomorphic to $T_{0}$.

We turn now to the discussion of the existence of a basis for a topological semigroup.

As we have seen in 5.2 and 5.3, not every compact semigroup has a basis. However, we also note that the semigroups of 5.2 and 5.3 are not reductive. The question then arises as to whether each compact reductive semigroup has a basis. We will answer this in the negative by presenting an example of a compact semilattice having no basis. Let us first proceed to demonstrate why one cannot expect the standard Zorn's lemma argument to work.

If $S$ is a semigroup, and $n$ is a positive integer, let $S^{n}=\left\{x \in S: x=a_{1} a_{2}\right.$ $\cdots a_{n}$ for $\left.a_{1}, a_{2}, \ldots, a_{n} \in S\right\}$. Note that $S^{n}$ is an ideal of $S$, and if $S$ is a compact topological semigroup, then $S^{n}$ is closed and hence compact. We remark also that if $S$ is [left] right reductive, then $S^{n}$ is [left] right reductive. To see this for the left reductive case, suppose that $S$ is left reductive and $a \neq b$ in $S^{n}$. Then there exists $t_{n} \in S$ such that $t_{n} a \neq t_{n} b$, and hence there exists $t_{n-1}$ $\in S$ such that $t_{n-1} t_{n} a \neq t_{n-1} t_{n} b$. Continuing recursively, we obtain $t_{1}, t_{2}$, $\cdots, t_{n} \in S$ such that for $t=t_{1} t_{2} \cdots t_{n}$, we have $t a \neq t b$. Since $t \in S^{n}$, we conclude that $S^{n}$ is left reductive. It follows trivially that if $S$ is reductive, then $S^{n}$ is reductive. An additional observation which we will employ is that if $\omega$ is a bitranslation of $S$, then $\omega \mid S^{n}$ is a bitranslation of $S^{n}$. To see that $\omega x \in S^{n}$ for $x \in S^{n}$, write $x=x_{1} x_{2} \cdots x_{n}$, where $x_{1}, x_{2}, \ldots, x_{n} \in S$. Then $\omega x=$ $\omega\left(x_{1} x_{2} \cdots x_{n}\right)=\left(\omega x_{1}\right) x_{2} \cdots x_{n}$ which is in $S^{n}$.

5.16. Proposition. Let $S$ be a reductive semigroup and let $n$ be a positive integer. Then $S^{n}$ is a determination subset of $S$.

Proof. Let $\omega$ and $\omega^{\prime}$ be bitranslations of $S$ such that $\omega\left|S^{n}=\omega^{\prime}\right| S^{n}$ and let $x \in S$. Then for each $t_{2}, \ldots, t_{n} \in S$, we have $(\omega x) t_{2} \cdots t_{n}=$ $\omega\left(x t_{2} \cdots t_{n}\right)=\omega^{\prime}\left(x t_{2} \cdots t_{n}\right)=\left(\omega^{\prime} x\right) t_{2} \cdots t_{n}$. With $n-1$ applications of right reductivity, we obtain that $\omega x=\omega^{\prime} x$, and similarly $x \omega=x \omega^{\prime}$ using teft reductivity. It follows that $\omega=\omega^{\prime}$.

Although the following proposition is not pertinent to our discussion of the existence of a basis, we present it here as a computational algorithm and note that it provides an alternate method of dealing with the example of 5.12.

5.17. Proposition. Let $S$ be a compact reductive topological semigroup 
and let $n$ be a positive integer. Then $\omega \mapsto \omega \mid S^{n}$ is an embedding of $\Omega_{p}(S)$ into $\Omega_{p}\left(S^{n}\right)$.

Proof. It is straightforward to verify that $\omega \mapsto \omega \mid S^{n}$ is a continuous homomorphism of $\Omega_{p}(S)$ into $\Omega_{p}\left(S^{n}\right)$. The injectivity follows from 5.16 , and the conclusion from the fact that $\Omega_{p}(S)$ is compact and $\Omega_{p}\left(S^{n}\right)$ is Hausdorff.

We demonstrate that the intersection of determination subsets of a compact reductive topological semigroup need not be a determination subset in the following simple example:

5.18. ExAmple. Let $S=[0,1 / 2]$ with the usual multiplication and topology, and let $N$ denote the set of all positive integers. Then $S$ is a compact reductive semigroup, and $S^{n}$ is a determination subset of $S$ for each $n \in N$.

Observe however, that $\bigcap\left\{S^{n}\right\}_{n \in N}=\{0\}$, and $\{0\}$ is not a determination subset of $S$, since $\omega 0=0 \omega=0$ for each $\omega \in \Omega_{p}(S)$.

We now present our example of a compact semilattice with no basis.

5.19. EXAMPLE. Let $M=[0,1]$ with min multiplication, $T=\{(a, b) \in$ $M \times M: a+b \leqslant 1\}$ with coordinate multiplication, $I=\{(a, b) \in T: a=0$ or $b=0\}, S=T / I$, and $\eta: T \rightarrow S$ the natural homomorphism. Let $e=(0,1)$ and $f=(1,0)$ in $T$. We visualize $T$ and $S$ in the following illustration:
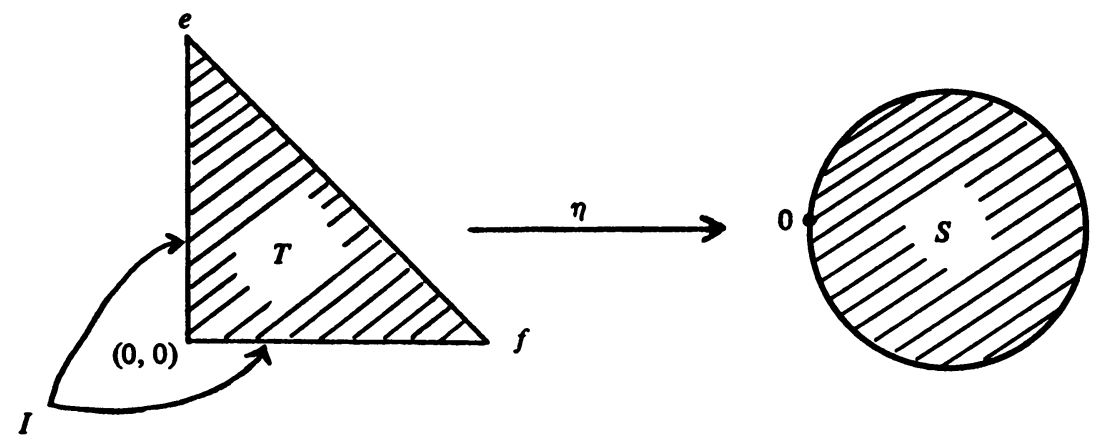

Let $P$ denote the subsemilattice $T$ of $T$.

We first show that a subset $D$ of $P$ is a separating subset of $P$ if and only if $D$ contains sequences $\left\{e_{n}\right\} \rightarrow e$ and $\left\{f_{n}\right\} \rightarrow f$ (in $T$ ).

For the necessity, let $D$ be a subset of $P$ containing no sequence which converges to $e$. Let $U$ be an open subset of $T$ of the form

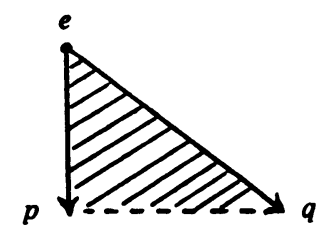

such that $U \cap D=\varnothing$. Fix distinct points $x$ and $y$ in $U \cap P$ which lie on the same vertical line. Then, as only points of $T$ which separate $x$ from $y$ lie in $U$, 
no point of $D$ separates $x$ and $y$, and hence $D$ is not a separating subset of $P$. Similarly, if $D$ contains no sequence converging to $f$ (in $T$ ), then $D$ is not a separating subset of $P$.

For the sufficiency, let $D$ contain sequences $\left\{e_{n}\right\} \rightarrow e$ and $\left\{f_{n}\right\} \rightarrow f$ and fix $x \neq y$ in $P$. Then $\left\{e_{n} x\right\} \rightarrow e x,\left\{e_{n} y\right\} \rightarrow e y,\left\{f_{n} x\right\} \rightarrow f x$, and $\left\{f_{n} y\right\} \rightarrow f y$. Since $\{e, f\}$ is a separating subset of $T$, either $e x \neq e y$ or $f x \neq f y$. It follows that either $e_{n} x \neq e_{n} y$ or $f_{n} x \neq f_{n} y$ for some positive integer $n$, so that $D$ is a separating subset.

Now, it is trivial to see that $P$ has no minimal separating subset.

If $B$ were a basis for $S$, then, according to $5.1, B$ would be a minimal separating subset of $S$, and $D=\eta^{-1}(B)$ would be a minimal separating subset of $P$, since clearly $\eta(I)=0 \notin B$. We conclude that $S$ has no basis.

The absence of a basis for $S$ does not prevent our discovering the structure of its translational hull. We shall use the next algorithm to perform that computation.

A Brouwerian lattice can be defined to be a complete lattice $(S, V, \wedge)$ such that for each $A \subset S$ and each $t \in S$, we have $t \wedge \sup A=\sup (t \wedge A)$.

5.20. Proposition. Let $(S, \vee, \wedge)$ be a Brouwerian lattice and let I be an $\wedge$-ideal of the semilattice $(S, \wedge)$. Then $\Omega(I, \wedge)$ is isomorphic to $(S \wedge b, \wedge)$, where $b=\sup I$.

Proof. Define $\alpha: S \wedge b \rightarrow \Omega(I, \wedge)$ by $\alpha(x)=\omega_{x} \mid I$ for each $x \in S \wedge b$, and define $\beta: \Omega(I, \wedge) \rightarrow S \wedge b$ by $\beta(\omega)=$ sup $\omega I$. From [10, p. 296], we have that $\alpha$ is a homomorphism. We will show that $\beta \circ \alpha$ is the identity map on $S \wedge b$, and that $\alpha \circ \beta$ is the identity map on $\Omega(I, \wedge)$.

To see that $\beta \circ \alpha$ is the identity map on $S \wedge b$, let $a \in S \wedge b$. Then $\beta \alpha(a)=\beta\left(\omega_{a} I\right)=\sup \omega_{a} I=\sup (a \wedge I)=a \wedge \sup I=a \wedge b=a$, so that $\beta \alpha$ is the identity map on $S \wedge b$.

To establish that $\alpha \beta$ is the identity map on $\Omega(I, \wedge)$, let $\omega \in \Omega(I, \wedge)$. Then $\alpha \beta(\omega)=\alpha(\sup \omega I)=\omega_{\text {sup } \omega I} \mid I=\omega$, since for $t \in I$, we have $\left(\omega_{\text {sup } \omega I} \mid I\right) t=$ $\sup \omega I \wedge t=\sup (\omega I \wedge t)=\sup \omega(I \wedge t)=\sup (I \wedge \omega t)=\sup I \wedge \omega t=\omega t$.

\subsection{Corollary. Let $(S, \wedge)$ be a compact topological semilattice} which is algebraically a distributive lattice and let $I$ be a closed $\wedge$-ideal of $S$. Then $\Omega_{p}(I, \wedge)$ is topologically isomorphic to $(S \wedge b, \wedge)$, where $b=\sup I$.

Proof. The isomorphism $\alpha: S \wedge b \rightarrow \Omega_{p}(I, \wedge)$ defined by $\alpha(x)=$ $\omega_{x} W$ (as in the proof of 5.20) is continuous and closed, and hence is a topological isomorphism.

In reference to 5.19 , note that the compact semilattice $T$ is a $\Lambda$-ideal of the compact lattice $M \times M$ and that sup $T$ is the identity of $M \times M$, so that $\Omega_{p}(T)$ is topologically isomorphic to $M \times M$. Similarly, the compact semi- 
lattice $S$ is an ideal of the compact lattice $(M \times M) / I$ and sup $S$ is the identity of $(M \times M) / I$, so that from 5.21 , we conclude that $\Omega_{p}(S)$ is topologically isomorphic to $(M \times M) / I$.

The process of determining the continuous bitranslations of a given semitopological semigroup $S$ is frequently complicated by restrictions on the images of elements of $S$ under bitranslations. For example, if $e$ is an idempotent of $S$ and $\omega$ is a bitranslation of $S$, then $\omega e \in S e$. Thus unlike vector space theory, one cannot generally assign images to basis elements and expect to determine a bitranslation. However, one can approach the problem by elimination of those image assignments which do not give bitranslations. We hasten to add that this technique is not always feasible, but also point out that it is feasible for some examples that have no basis to consider certain distinguished elements. For example, it is usually fruitful to consider the possible images of the generator of a compact monothetic semigroup or of a principal ideal of a compact solenoidal semigroup.

We close this section with some remarks pertaining to the algebraic analogues of some of the results in this section. Observe that if $S$ is a reductive semigroup, which admits the structure of a compact semitopological semigroup, then as each bitranslation of $S$ is continuous, the algebraic translational hull is algebraically the same as $\Omega_{p}(S)$. This gives us obvious algebraic analogues of $5.1,5.4,5.5,5.11,5.13,5.16$, and 5.17.

\section{REFERENCES}

O. R. Arens and J. Dugundji, Topologies for function spaces, Pacific J. Math. 1 (1951), 5-31. MR 13, 264.

1. T. T. Bowman, The semilattice of left translations of a compact semilattice, Notices Amer. Math. Soc. 20 (1973), p. A-673. Abstract \#709-G16.

2. F. T. Christoph, Jr., Ideal extensions of topological semigroups, Canad. J. Math. 22 (1970), 1168-1175. MR 42 \#3216.

3. A. H. Clifford and G. B. Preston, The algebraic theory of semigroups. Vol. I, Math. Surveys, no. 7, Amer. Math. Soc., Providence, R. I., 1961. MR 24 \#A2627.

4. M. Friedberg, On compactifying semigroups, Semigroup Forum 10 (1975), 39-54.

5. J. A. Hildebrant and J. D. Lawson, Embedding in compact uniquely divisible semigroups, Semigroup Forum 4 (1972), 295-300. MR 46 \#3651.

6. - The Bohr compactification of a dense ideal in a topological semigroup, Semigroup Forum 6 (1973), 86-92.

7. K. H. Hofmann and P. S. Mostert, Elements of compact semigroups, Merrill, Columbus, Ohio, 1966. MR 35 \#285.

8. J. L. Kelley, General topology, Van Nostrand, Princeton, N. J., 1955. MR 16, 1136.

9. J. D. Lawson, Joint continuity in semitopological semigroups, Illinois J. Math. 18 (1974), 275-285. MR 49 \#454.

10. M. Petrich, The translational hull in semigroups and rings, Semigroup Forum 1 (1970), 283-360. MR 42 \#1919.

11. - Introduction to semigroups, Merrill, Columbus, Ohio, 1973.

DEPARTMENT OF MATHEMATICS, LOUISIANA STATE UNIVERSITY, BATON ROUGE, LOUISIANA 70803 